\title{
Electric field stabilization of viscous liquid layers coating the underside of a surface
}

\author{
Thomas G. Anderson \\ Department of Computing \& Mathematical Sciences, California Institute of Technology, \\ Pasadena, California 91125, USA
}

Radu Cimpeanu* and Demetrios T. Papageorgiou

Department of Mathematics, Imperial College London, SW7 2AZ London, United Kingdom

Peter G. Petropoulos

Department of Mathematical Sciences, New Jersey Institute of Technology, Newark, New Jersey 07102, USA

(Received 9 January 2017; published 11 May 2017)

\begin{abstract}
We investigate the electrostatic stabilization of a viscous thin film wetting the underside of a horizontal surface in the presence of an electric field applied parallel to the surface. The model includes the effect of bounding solid dielectric regions above and below the liquid-air system that are typically found in experiments. The competition between gravitational forces, surface tension, and the nonlocal effect of the applied electric field is captured analytically in the form of a nonlinear evolution equation. A semispectral solution strategy is employed to resolve the dynamics of the resulting partial differential equation. Furthermore, we conduct direct numerical simulations (DNS) of the Navier-Stokes equations using the volume-of-fluid methodology and assess the accuracy of the obtained solutions in the long-wave (thin-film) regime when varying the electric field strength from zero up to the point when complete stabilization occurs. We employ DNS to examine the limitations of the asymptotically derived behavior as the liquid layer thickness increases and find excellent agreement even beyond the regime of strict applicability of the asymptotic solution. Finally, the asymptotic and computational approaches are utilized to identify robust and efficient active control mechanisms allowing the manipulation of the fluid interface in light of engineering applications at small scales, such as mixing.
\end{abstract}

DOI: 10.1103/PhysRevFluids.2.054001

\section{INTRODUCTION}

Rayleigh-Taylor instabilities of liquid layers have been studied by numerous authors both theoretically and experimentally. Linear stability analysis and two-dimensional nonlinear computations can be found in Refs. [1-3], for example, where it is found that dripping transitions take place for sufficiently thick layers with fingers of heavy fluid penetrating lighter fluid. In the case of films thinner than the capillary length $(\sigma / \rho g)^{1 / 2}$ (here $\sigma$ is the surface tension coefficient, $\rho$ is the density, and $g$ the gravitational acceleration), dripping does not take place, but instead drops are formed that are connected by slowly draining thinning regions. The thin-film models describing such phenomena were derived and studied by Yiantsios and Higgins [1], and interestingly the equations are identical to the axisymmetric capillary film draining equations studied by Hammond [4] that are driven by capillary instabilities in the absence of gravity. The dynamics of drop formation become much more intricate on longer domains that can allow drops to move within the domain, as shown by accurate computations and analysis by Lister et al. [5]. Motivated by the experiments and weakly nonlinear analyses of Limat and co-workers [6,7], the one-dimensional patterns studied in Ref. [5]

*Corresponding author: radu.cimpeanu@imperial.ac.uk 
were extended to two-dimensional ones by Lister et al. [8], who found behavior such as droplet coalescence or bouncing.

The stabilization of the gravity-driven Rayleigh-Taylor instability has also been considered by several authors. Babchin et al. [9] showed that a nonlinear saturation is possible in the presence of a constant background shear; this is analyzed for a two-fluid Couette flow with a thin lighter film beneath a heavier upper fluid. Stabilization was also demonstrated by Halpern and Frenkel [10] for cases when the shear arises due to zero-mean time-periodic oscillations of the upper plate of a two-fluid Couette flow. Stabilization was also predicted theoretically in Ref. [11] for liquid films coating the outside surface of a horizontal cylinder performing time-periodic oscillations along its axis.

The present study considers the utilization of electric fields in the linear and nonlinear manipulation of the Rayleigh-Taylor instability and its ultimate stabilization. It is known from early work by Melcher [12,13] that a tangential electric field has a stabilizing effect on interfacial waves and the use of electric fields in linearly stabilizing Rayleigh-Taylor instabilities was considered by Eldabe [14] and more recently by Joshi et al. [15]. Several nonlinear studies and direct numerical simulations have been carried out. Barannyk et al. $[16,17]$ used an symptomatic theory valid for thin layers to derive reduced models in the case of inviscid dielectric fluids; they show that above a critical value of the applied electric field the flow can be completely stabilized, whereas at subcritical values the fluid layer thins and the interface touches the wall in finite time. The mathematical structures of such touching singularities (including self-similar forms and their confirmation via numerical computations) were explored in detail in Ref. [17], in both the presence and absence of surface tension. If surface tension is present the local geometry of the touching interface maintains bounded gradients but unbounded curvature (a corner singularity) with electric field effects being of higher order; for electrified flows with zero surface tension the touching singularities are worse in the sense that cusps form with interfacial gradients blowing up locally. One of the objectives of the present study is to include viscosity and also more realistic electric field configurations. We also note that direct numerical simulations for electrified Rayleigh-Taylor flows that are unbounded in the vertical direction were carried out by Cimpeanu et al. [18], who find complete stabilization of finite wavelength perturbations and in addition quantify the effect of the field on the formation and dynamics of finger formation and propagation of the heavier fluid into the lighter one.

The structure of the paper is as follows. Section II describes the mathematical model and carries out an asymptotic analysis to derive an evolution equation for the dynamics of thin films. Section III presents computations based on the model equation and describes the effect of the electric field on the solutions. Section IV presents direct numerical computations of the problem valid for arbitrary thickness fluid layers and arbitrary Reynolds numbers. A direct comparison between the asymptotic and numerical solutions is also presented. Section V uses the results to suggest an application of utilizing electric fields to produce controlled interfacial oscillations that do not rupture the layer and do not produce dripping. We conclude with a discussion in Sec. VI.

\section{MATHEMATICAL MODEL}

\section{A. Governing equations}

We consider two superposed immiscible fluid layers placed in the gap between two solid dielectric slabs of infinite vertical extent (see the schematic in Fig. 1); the flow is assumed to be two dimensional. A horizontal electric field driven by lateral electrodes far away is assumed to be present in both the fluids and the solid dielectric bounding slabs as shown in the schematic. The dynamics are driven by the competition between gravitational, surface tension, and electric field effects, with the latter two acting to stabilize Rayleigh-Taylor instability in an initially quiescent flow.

The overhanging layer (region 2) wetting the underside of the upper slab is a dielectric Newtonian liquid with constant density $\rho_{2}^{*}$, viscosity $\mu_{2}^{*}$, and permittivity $\epsilon_{2}^{*}$ and lies in $h^{*} \leqslant y^{*} \leqslant h_{u}^{*}$; here $y^{*}=h^{*}\left(x^{*}, t^{*}\right)$ is the liquid-liquid interface and $y^{*}=h_{u}^{*}$ is the position of the flat lower boundary 
ELECTRIC FIELD STABILIZATION OF VISCOUS ...

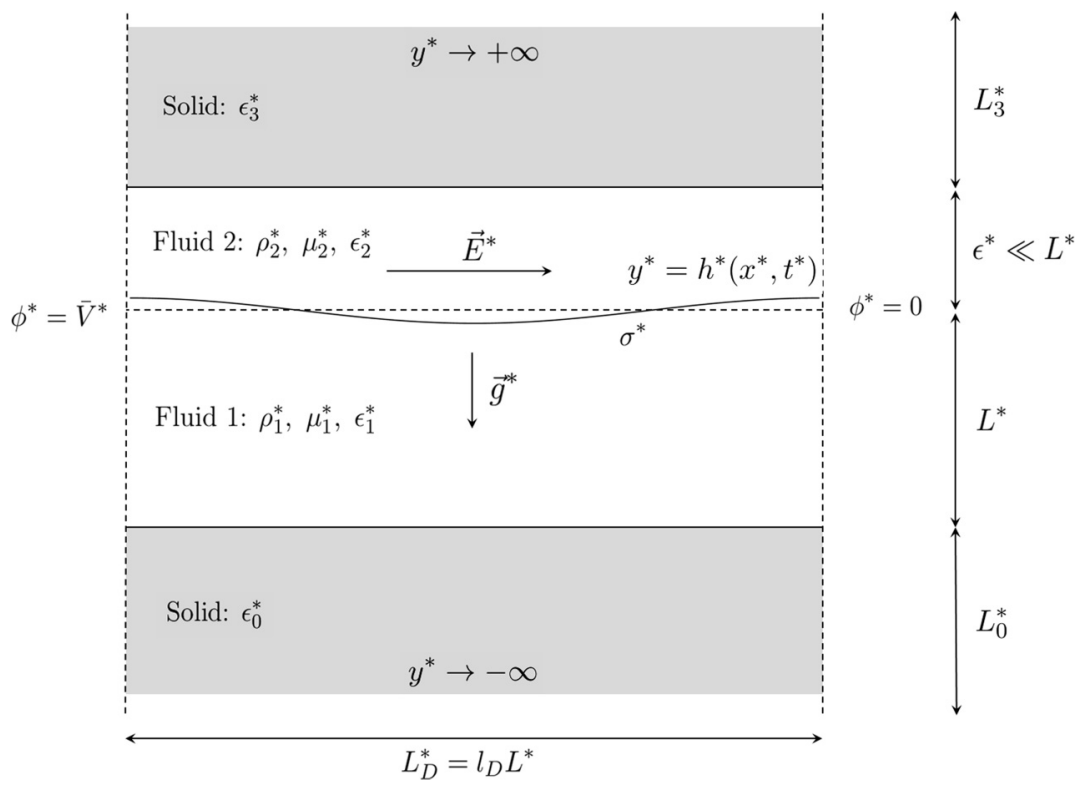

FIG. 1. Schematic of the problem with fluid 2 lying above fluid 1 and separated by the interface $y^{*}=$ $h^{*}\left(x^{*}, t^{*}\right)$. A uniform electric field of size $E^{*}=\bar{V}^{*} / L_{D}^{*}$ is applied horizontally as shown.

of the upper solid slab region $3, y^{*}>h_{u}^{*}$. The lower dielectric fluid region 1 in $0 \leqslant y^{*} \leqslant h^{*}$ is much less dense and viscous and is hydrodynamically passive to leading order (the negligible physical properties simplify the governing equations to describe a region of zero velocities and uniform pressure); its electrical permittivity is $\epsilon_{1}^{*}$. This is bounded below by another solid slab denoted by region 0 and lying in $y^{*}<0$. The dielectric permittivities in regions 0 and 3 are $\epsilon_{0}^{*}$ and $\epsilon_{3}^{*}$, respectively. In addition, surface tension is present with constant coefficient $\sigma^{*}$ and gravity is acting in the vertical direction with constant acceleration $g^{*}$. The velocity field in the upper liquid layer is $\mathbf{u}_{2}^{*}=\left(u_{2}^{*}, v_{2}^{*}\right)$ and the pressures in regions 1 and 2 are $p_{1,2}^{*}$. The electrodes are kept at constant voltage potentials $\phi^{*}=\bar{V}^{*}$ on the left and $\phi^{*}=0$ on the right as shown in Fig. 1, resulting in a uniform horizontal electric field of magnitude $E^{*}=\bar{V}^{*} / L_{D}^{*}$ in the undisturbed configuration.

The Navier-Stokes and continuity equations hold in region 2 ,

$$
\begin{gathered}
\rho_{2}^{*}\left(\mathbf{u}_{2 t}^{*}+\mathbf{u}_{2}^{*} \cdot \nabla \mathbf{u}_{2}^{*}\right)=-\nabla p_{2}^{*}+\mu_{2}^{*} \Delta \mathbf{u}_{2}^{*}-\rho_{2}^{*} g^{*} \mathbf{j}, \\
\nabla \cdot \mathbf{u}_{2}^{*}=0,
\end{gathered}
$$

and voltage potentials $\phi_{0,1,2,3}^{*}$ are present in each region producing an electric field $\mathbf{E}_{i}^{*}=-\nabla \phi_{i}^{*}$ there. In the absence of volume charges, the voltage potentials satisfy Laplace equations in each region (this follows from the electrostatic limit of Maxwell's equations)

$$
\nabla^{2} \phi_{0,1,2,3}^{*}=0,
$$

where $\nabla^{2}=\partial_{x^{*}}^{2}+\partial_{y^{*}}^{2}$. At the interface $y^{*}=h^{*}\left(x^{*}, t^{*}\right)$ we must impose the balance of normal and tangential stresses

$$
\left[\mathbf{n} \cdot \mathcal{T}^{*} \cdot \mathbf{n}\right]_{2}^{1}=\sigma^{*} \kappa, \quad\left[\mathbf{t} \cdot \mathcal{T}^{*} \cdot \mathbf{n}\right]_{2}^{1}=0,
$$

where $[(\cdot)]_{2}^{1}=(\cdot)_{1}-(\cdot)_{2}$ denotes the jump across the interface, $\mathbf{n}=\left(-h_{x^{*}}^{*}, 1\right) /\left(1+h_{x^{*}}^{* 2}\right)^{1 / 2}$ and $\mathbf{t}=\left(1, h_{x^{*}}^{*}\right) /\left(1+h_{x^{*}}^{* 2}\right)^{1 / 2}$ are the unit normal and tangent to the interface, respectively, and $\kappa=$ $h_{x^{*} x^{*}}^{*} /\left(1+h_{x^{*}}^{* 2}\right)^{3 / 2}$ is the interfacial curvature. The stress tensor $\mathcal{T}^{*}$ contains electric and fluid parts 
and is given by

$$
\mathcal{T}_{i j}^{*}=-p^{*} \delta_{i j}+\tilde{\mu}^{*}\left(\frac{\partial u_{i}^{*}}{\partial x_{j}^{*}}+\frac{\partial u_{j}^{*}}{\partial x_{i}^{*}}\right)+\epsilon^{*} \tilde{E}_{i}^{*} \tilde{E}_{j}^{*}-\frac{1}{2} \epsilon^{*}\left|\mathbf{E}^{*}\right|^{2} \delta_{i j},
$$

where it is understood that the appropriate subscript is used in different regions. As a result of the fluids being perfect dielectrics with constant permittivities, there are no charges present in the flow and hence the Lorentz force has no contribution in the momentum conservation equation (1). Instead, the jump in Maxwell stresses manifests itself nonlinearly in the stress balance conditions (4). In addition, we have a kinematic condition

$$
v_{2}^{*}=h_{t^{*}}^{*}+u_{2}^{*} h_{x^{*}}^{*} \text { at } y^{*}=h^{*}\left(x^{*}, t^{*}\right),
$$

and no-slip conditions at the solid wall,

$$
u_{2}^{*}=v_{2}^{*}=0 \quad \text { at } y^{*}=h_{u}^{*} .
$$

The interfacial boundary conditions for the electric field are Gauss's law and continuity of the tangential component

$$
\left[\epsilon^{*} \mathbf{E}^{*} \cdot \mathbf{n}\right]_{i}^{i+1}=0, \quad\left[\mathbf{t} \cdot \mathbf{E}^{*}\right]_{i}^{i+1}=0, \quad i=0,1,2 .
$$

The second condition is equivalent to

$$
\left[\phi^{*}\right]_{0}^{1}=0, \quad\left[\phi^{*}\right]_{1}^{2}=0, \quad\left[\phi^{*}\right]_{2}^{3}=0 .
$$

We nondimensionalize velocities using the scale $U^{*}=\sqrt{g^{*} L^{*}}$ so as to retain the gravity-driven Rayleigh-Taylor instability. The lower fluid layer height $L^{*}$ is used as a reference length scale, time is scaled by $L^{*} / \sqrt{g^{*} L^{*}}$, and pressure is scaled according to $p^{*} \sim \rho_{2}^{*} U^{* 2} \sim \rho_{2}^{*} g^{*} L^{*}$. The voltage potentials are nondimensionalized using $V_{0}^{*}$. (In what follows we use the same symbols for dimensionless variables, but they are unstarred.) The following dimensionless groups emerge from the manipulation of the equations and boundary conditions

$$
\tilde{g}=\frac{g^{*} L^{*}}{U^{* 2}} \equiv 1, \quad \tilde{\mu}=\frac{\mu_{2}^{*}}{\rho_{2}^{*} L^{*}\left(g^{*} L^{*}\right)^{1 / 2}}, \quad W_{e}=\frac{\sigma^{*}}{\rho_{2}^{*} g^{*} L^{* 2}}, \quad E_{b}=\frac{\epsilon_{1}^{*} V_{0}^{* 2}}{\rho_{2}^{*} g^{*} L^{* 3}} .
$$

These represent the unit inverse square Froude number $\tilde{g}$, an inverse Reynolds number $\tilde{\mu}$, an inverse Weber number $W_{e}$, and an electric dimensionless group $E_{b}$. Note that we have used quantities in the upper fluid layer 2 as reference for hydrodynamic values, while the permittivity $\epsilon_{1}^{*}$ of region 1 is selected as reference, leading to the relevant relative permittivity ratios

$$
\epsilon_{B}=\epsilon_{0}^{*} / \epsilon_{1}^{*}, \quad \epsilon_{p}=\epsilon_{2}^{*} / \epsilon_{1}^{*}, \quad \epsilon_{T}=\epsilon_{3}^{*} / \epsilon_{1}^{*} .
$$

Hence, the dimensionless Navier-Stokes equations in region 2 become

$$
\begin{gathered}
\mathbf{u}_{t}+(\mathbf{u} \cdot \nabla) \mathbf{u}=-\nabla p_{2}+\tilde{\mu} \Delta \mathbf{u}-\tilde{g} \mathbf{j}, \\
\nabla \cdot \mathbf{u}=0,
\end{gathered}
$$

where we have removed the subscript 2 since the bottom layer region 1 is hydrodynamically passive. The normal and tangential stress balances at $y=h(x, t)$ [recall Eq. (4)] reduce to

$$
\begin{gathered}
-\left(1+h_{x}^{2}\right)\left(p_{1}-p_{2}\right)+2 \tilde{\mu} h_{x}\left(v_{x}+u_{y}\right)-2 \tilde{\mu}\left(v_{y}+h_{x}^{2} u_{x}\right)-2 h_{x} E_{b} \frac{\partial \phi_{1}}{\partial x} \frac{\partial \phi_{1}}{\partial y} \\
+2 \epsilon_{p} E_{b} h_{x} \frac{\partial \phi_{2}}{\partial x} \frac{\partial \phi_{2}}{\partial y}+\frac{1}{2} E_{b}\left(h_{x}^{2}-1\right)\left[\left(\frac{\partial \phi_{1}}{\partial x}\right)^{2}-\left(\frac{\partial \phi_{1}}{\partial y}\right)^{2}\right] \\
054001-4
\end{gathered}
$$




$$
\begin{aligned}
-\frac{1}{2} \epsilon_{p} E_{b}\left(h_{x}^{2}-1\right)\left[\left(\frac{\partial \phi_{2}}{\partial x}\right)^{2}-\left(\frac{\partial \phi_{2}}{\partial y}\right)^{2}\right] & =W_{e} \frac{h_{x x}}{\left(1+h_{x}^{2}\right)^{1 / 2}}, \\
2 h_{x}\left(u_{x}-v_{y}\right)-\left(1-h_{x}^{2}\right)\left(u_{y}+v_{x}\right) & =0 .
\end{aligned}
$$

Finally, the kinematic condition reads

$$
v=h_{t}+u h_{x} \quad \text { at } y=h(x, t) .
$$

The Laplace equations ( 3 ) for the voltages are unchanged and so are the voltage continuity conditions (9). The latter are simple at the fixed solid boundaries $y=0$ and $y=h_{u}$, while at the deforming interface voltage continuity takes the form

$$
\frac{\partial \phi_{2}}{\partial x}+h_{x} \frac{\partial \phi_{2}}{\partial y}=\frac{\partial \phi_{1}}{\partial x}+h_{x} \frac{\partial \phi_{1}}{\partial y} \quad \text { at } y=h(x, t) .
$$

Finally, the Gauss law conditions (continuity of the electric displacement field) become

$$
\begin{gathered}
\epsilon_{B} \frac{\partial \phi_{0}}{\partial y}=\frac{\partial \phi_{1}}{\partial y} \quad \text { at } y=0, \\
-h_{x} \epsilon_{p} \frac{\partial \phi_{2}}{\partial x}+\epsilon_{p} \frac{\partial \phi_{2}}{\partial y}=-h_{x} \frac{\partial \phi_{1}}{\partial x}+\frac{\partial \phi_{1}}{\partial y} \quad \text { at } y=h(x, t), \\
\epsilon_{p} \frac{\partial \phi_{2}}{\partial y}=\epsilon_{T} \frac{\partial \phi_{3}}{\partial y} \quad \text { at } y=h_{u} .
\end{gathered}
$$

Using bars to denote the undisturbed state characterized by a flat interface, the above system admits the exact solution

$$
\bar{h}=1, \quad \bar{u}=\bar{v}=0, \quad \bar{\phi}_{0,1,2,3}=x .
$$

This solution is unstable and in what follows we present a nonlinear theory valid for thin fluid layers and describe the spatiotemporal dynamics under the action of electric fields. Direct numerical simulations are considered later (see Sec. IV).

\section{B. Evolution equation for thin liquid films}

Assume that the upper liquid layer region 2 has mean thickness $\epsilon \ll 1$ so that in its undisturbed state it is bounded between $y=\bar{h}=1$ and $y=h_{u}=1+\epsilon$. We consider interfacial deflections that have order one wavelengths and order $\epsilon$ amplitudes, i.e., fully nonlinear in the sense that they scale with the fluid layer thickness. We write the interfacial position as

$$
y=h(x, t)=1+\epsilon \tilde{h}(x, t), \quad \tilde{h}(x, t)=O(1) .
$$

It is useful to introduce a stretched inner coordinate $\eta$ given by $y=1+\epsilon-\epsilon \eta$ so that $\partial_{y} \rightarrow-\epsilon^{-1} \partial_{\eta}$. The order $\epsilon$ deflection produces equivalent perturbations to the voltage potentials and the pressure jump contribution due to surface tension. The appropriate expansions are therefore

$$
p_{1}=\bar{p}_{1}, \quad p_{2}=\bar{p}_{2}+1-y+\epsilon \tilde{p}+\cdots, \quad \phi_{j}=x+\epsilon \tilde{\phi}_{j}^{(0)}+\epsilon^{2} \tilde{\phi}_{j}^{(1)}+\cdots,
$$

where $j=0,1,2,3$ and $\bar{p}_{1}$ and $\bar{p}_{2}$ are constants; in fact, $\bar{p}_{2}-\bar{p}_{1}=E_{b}\left(1-\epsilon_{p}\right) / 2$ as follows from (14) for the base state solution. Note also that the term $1-y$ in the expression for $p_{2}$ is the hydrostatic contribution. The Navier-Stokes equations are used next to obtain the scalings for the velocities required for the viscous terms to balance pressure. Under the assumption that the Reynolds number $\operatorname{Re}=\tilde{\mu}^{-1}=O(1)$, we find

$$
u=\epsilon^{3} \tilde{u}_{0}+\epsilon^{4} \tilde{u}_{1}+\cdots, \quad v=\epsilon^{4} \tilde{v}_{0}+\epsilon^{5} \tilde{v}_{1}+\cdots,
$$


with the scaling for $v$ being a direct consequence of mass conservation. Finally, the relevant time scale for the dynamics is found from the kinematic condition, which to leading order reads

$$
\epsilon^{3} \tilde{v}_{0}=\tilde{h}_{t}+\epsilon^{3} \tilde{u}_{0} \tilde{h}_{x} \quad \text { at } \eta=1-\tilde{h},
$$

which leads to a slow time scale

$$
\tau=\epsilon^{3} \tilde{\mu}^{-1} t
$$

After solving for $u_{0}$ and $v_{0}$ from the leading-order contributions of the Navier-Stokes equations and substituting into (25), the following evolution equation for the interface is found:

$$
\tilde{h}_{\tau}+\frac{\partial}{\partial x}\left[\tilde{p}_{x} \frac{(1-\tilde{h})^{3}}{3}\right]=0 .
$$

It remains to determine $\tilde{p}_{x}$ at the interface in terms of $\tilde{h}$ to obtain a closed system. This is achieved by expanding the normal stress balance condition (14) to obtain, at leading order,

$$
\tilde{p}=\tilde{h}+W_{e} \tilde{h}_{x x}+E_{b}\left(\left.\tilde{\phi}_{1 x}^{(0)}\right|_{y=1}-\left.\epsilon_{p} \tilde{\phi}_{2 x}^{(0)}\right|_{\eta=1-\tilde{h}}\right) .
$$

Note that in this expression we have used the solution (35) derived below, which ensures that the Maxwell stress contribution $\phi_{2 y}^{2}$ to (14) is of order $\epsilon^{2}$ at most and does not contribute to (28). The horizontal electric field terms $\tilde{\phi}_{1 x}^{(0)}$ and $\tilde{\phi}_{2 x}^{(0)}$ need to be determined by solving the system (3) and (20) and their appropriate boundary conditions. The voltage potential in region 1 can be readily solved in Fourier space:

$$
\frac{d^{2} \widehat{\tilde{\phi}_{1}^{(0)}}}{d y^{2}}-k^{2} \widehat{\tilde{\phi}_{1}^{(0)}}=0 \Rightarrow \widehat{\phi_{1}^{(0)}}=\alpha(k) \cosh (k y)+\beta(k) \sinh (k y),
$$

with $\alpha(k)$ and $\beta(k)$ to be found (carets denote Fourier transforms in the usual way). The potentials $\tilde{\phi}_{1}$ and $\tilde{\phi}_{2}$ are linked through (19), which on use of the expansions (22) and (23) becomes

$$
-\left.\epsilon_{p} \tilde{\phi}_{2 \eta}^{(0)}\right|_{\eta=1-\tilde{h}}-\epsilon \epsilon_{p}\left(\tilde{h}_{x}+\left.\tilde{\phi}_{2 \eta}^{(1)}\right|_{\eta=1-\tilde{h}}\right)+O\left(\epsilon^{2}\right)=-\epsilon\left(\tilde{h}_{x}-\left.\tilde{\phi}_{1 y}^{(0)}\right|_{y=1}\right)+O\left(\epsilon^{2}\right) .
$$

In the thin-film region 2, the Laplace equation becomes $\epsilon^{2} \phi_{2 x x}+\phi_{2 \eta \eta}=0$ and from the expansions (23) we have

$$
\tilde{\phi}_{2 \eta \eta}^{(0)}=0, \quad \tilde{\phi}_{2 \eta \eta}^{(1)}=0
$$

whose solutions are

$$
\tilde{\phi}_{2}^{(0)}=A_{0}(x, \tau) \eta+B_{0}(x, t), \quad \tilde{\phi}_{2}^{(1)}=A_{1}(x, \tau) \eta+B_{1}(x, t),
$$

with $A_{0}, A_{1}, B_{0}$, and $B_{1}$ to be found. An additional coupling to the fields in the slab regions 0 and 3 is present due to the voltage continuity conditions at $y=0,1+\epsilon$ and the Gauss laws (18) and (20). Following [19], we use the fact that the complex functions $\partial_{x} \phi_{j}-i \partial_{y} \phi_{j}$ with $j=0,3$ are analytic in their respective domains and apply Cauchy's theorem in regions 0 and 3 in turn. Imposing the voltage continuity conditions at the walls leads to the following nonlocal boundary conditions, written in unscaled form for the moment:

$$
\begin{gathered}
-\frac{\epsilon_{B}}{\pi} \text { P.V. } \int_{-\infty}^{+\infty} \frac{\phi_{1 x}\left(x^{\prime}, 0\right)}{x^{\prime}-x} d x^{\prime}=\left.\frac{\partial \phi_{1}}{\partial y}\right|_{y=0}, \\
\frac{\epsilon_{T}}{\pi} \text { P.V. } \int_{-\infty}^{+\infty} \frac{\phi_{2 x}\left(x^{\prime}, 1+\epsilon\right)}{x^{\prime}-x} d x^{\prime}=\left.\epsilon_{p} \frac{\partial \phi_{2}}{\partial y}\right|_{y=1+\epsilon},
\end{gathered}
$$


where P.V. denotes the principal value of the integral. Introducing the expansions (23) and the inner variable $\eta$ into (33b), we find at order 1 and order $\epsilon$, respectively,

$$
-\left.\epsilon_{p} \tilde{\phi}_{2 \eta}^{(0)}\right|_{\eta=0}=0, \quad \frac{\epsilon_{T}}{\pi} \mathrm{P} . \mathrm{V} . \int_{-\infty}^{\infty} \frac{\phi_{2 x}^{(0)}\left(x^{\prime}, \eta=0\right)}{x^{\prime}-x} d x^{\prime}=-\left.\epsilon_{p} \tilde{\phi}_{2 \eta}^{(1)}\right|_{\eta=0} .
$$

The first condition implies that $A_{0}(x, \tau)=0$ in Eq. (32) so that

$$
\tilde{\phi}_{2}^{(0)}=B_{0}(x, \tau)
$$

while the second condition yields

$$
\frac{\epsilon_{T}}{\pi} \text { P.V. } \int_{-\infty}^{\infty} \frac{B_{0 x}\left(x^{\prime}, \tau\right)}{x^{\prime}-x} d x^{\prime}=-\epsilon_{p} A_{1}(x, \tau) .
$$

Using solutions (32) and (35) in the Gauss law (30) gives

$$
\left.\tilde{\phi}_{1 y}^{(0)}\right|_{y=1}=\left(1-\epsilon_{p}\right) \tilde{h}_{x}-\epsilon_{p} A_{1}
$$

and substituting (29) and (36) and going into Fourier space we find

$$
k \alpha(k) \sinh (k)+k \beta(k) \cosh (k)=i k\left(1-\epsilon_{p}\right) \widehat{\tilde{h}}-\epsilon_{T}|k| \widehat{B_{0}} .
$$

Two more conditions connecting the unknowns $\alpha(k), \beta(k)$, and $\widehat{B_{0}}(k)$ arise from the order $\epsilon$ contributions of condition (33a) and continuity of voltage potentials across the fluid-air interface. These are

$$
\epsilon_{B}|k| \widehat{\phi_{1}^{(0)}}=\left.\frac{d \widehat{\phi_{1}^{(0)}}}{d y}\right|_{y=0},\left.\quad \widehat{\phi_{1}^{(0)}}\right|_{y=0}=\widehat{B_{0}}
$$

Solving (38) and (39) allows us to express $B_{0}$ in terms of $\tilde{h}$ :

$$
\widehat{B}(k)=i\left(1-\epsilon_{p}\right) \frac{k \cosh (k)+\epsilon_{B}|k| \sinh (k)}{\left(\epsilon_{B}+\epsilon_{T}\right)|k| \cosh (k)+\left(1+\epsilon_{B} \epsilon_{T}\right) k \sinh (k)} \widehat{\tilde{h}} .
$$

It also follows from the solutions just obtained that (28) takes the form $\tilde{p}=\tilde{h}+W_{e} \tilde{h}_{x x}+E_{b}(1-$ $\left.\epsilon_{p}\right) B_{0 x}$ and hence the evolution equation (27) becomes

$$
h_{t}+\frac{1}{3}\left[(1-h)^{3}\left(h_{x}+W_{e} h_{x x x}+E_{b}\left(1-\epsilon_{p}\right) B_{x x}\right)\right]_{x}=0,
$$

where for simplicity we have dropped the tilde decoration and $\tau$ has been replaced by $t$. The interface will touch the wall if $h \rightarrow 1$ from below. We describe our numerical solution of (41) in the Appendix.

Note that in the absence of the lower dielectric slab (region 0), the nonlocal expression (40) simplifies to a form that shares strong similarities with the corresponding term in the evolution equation previously derived by Tseluiko and Papageorgiou [20]. In the respective case the sign is shifted due to their destabilizing vertical electric field configuration, while the prefactor is also different, as here we include the influence of the upper solid region 3 through $\epsilon_{T}$. The same result can be retrieved by considering the small-wavelength (large $k$ ) limit of the present setup.

\section{NUMERICAL RESULTS FOR THE THIN-FILM EQUATION}

In order to gain a fundamental understanding of the competition of the modeled physical phenomena and provide a quantitative basis for subsequent nonlinear computations, we first conduct a linear study of the long-wave evolution equation (41), focusing on the effects of varying the electric field strength. Linearizing about $h \equiv 0$ and seeking solutions proportional to $\exp (\omega t+i k x)$ yields the dispersion relation

$$
\omega(k)=\frac{1}{3} k^{2}-\frac{1}{3} W_{e} k^{4}-\frac{1}{3}\left(1-\epsilon_{p}\right)^{2} E_{b} k^{3} \frac{k \cosh (k)+\epsilon_{B}|k| \sinh (k)}{k \sinh (k)\left(1+\epsilon_{B} \epsilon_{T}\right)+|k| \cosh (k)\left(\epsilon_{B}+\epsilon_{T}\right)} .
$$




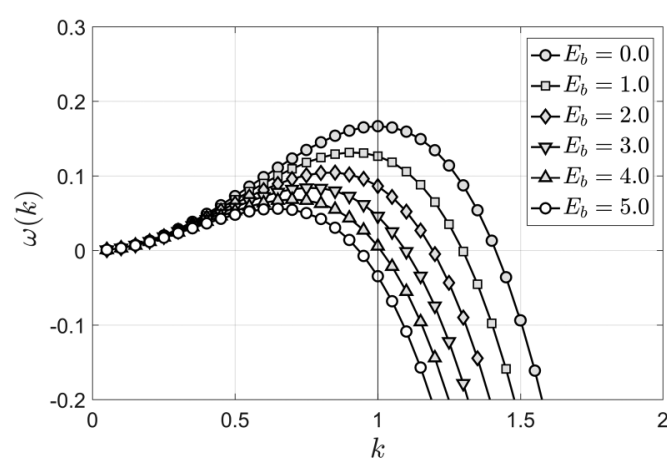

(a)

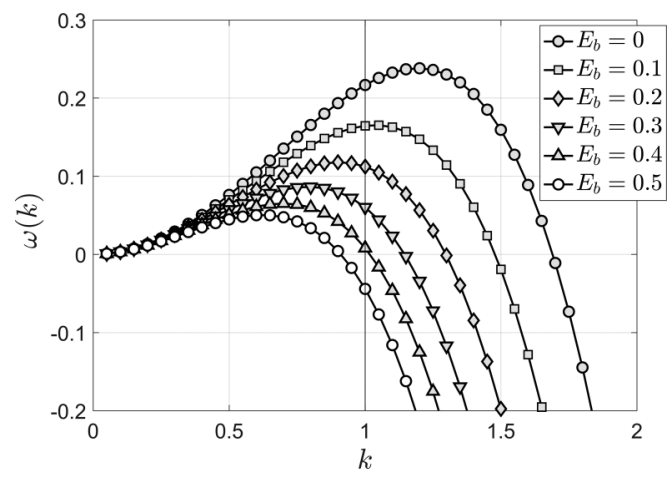

(b)

FIG. 2. Linear growth rates, as defined in Eq. (42), for two example cases, illustrating the stabilizing effect of the electric field with adjustable strength via the $E_{b}$ parameter: (a) $W_{e}=0.5, \epsilon_{p}=1.5$, and $\epsilon_{B}=\epsilon_{T}=1.0625$ and (b) $W_{e}=0.35, \epsilon_{p}=3.0$, and $\epsilon_{B}=\epsilon_{T}=1.5$.

The first term on the right-hand side is destabilizing and originates from the gravitational acceleration; the second term accounts for the stabilizing effect of surface tension, while the final term corresponds to the electric field. The five parameters in the system, $W_{e}, E_{b}, \epsilon_{p}, \epsilon_{B}$, and $\epsilon_{T}$, are selected as far as possible to correspond to realistic values that could be found in desktop experiments. Note that in many practical contexts we find that the bounding solid regions are fabricated from the same material, implying $\epsilon_{B}=\epsilon_{T}$ and thus reducing the number of parameters even further.

We consider two typical but distinct example cases: (a) $W_{e}=0.5, E_{b}$ varying from 0 to 5.0, $\epsilon_{p}=1.5$, and $\epsilon_{B}=\epsilon_{T}=1.0625$ and (b) $W_{e}=0.35, E_{b}$ varying from 0 to $0.5, \epsilon_{p}=3.0$, and $\epsilon_{B}=\epsilon_{T}=1.5$. The resulting growth rates $\mathcal{R}(\omega(k))$ are illustrated in Figs. 2(a) and 2(b). The anticipated behavior of long-wave instability over a finite number of unstable modes is observed, with long waves (small $k$ ) remaining unstable irrespective of the parameter values and short waves (large $k$ ) eventually stabilized by surface tension. Increasing $E_{b}$ has a stabilizing effect; the maximum growth rate and band of unstable waves decrease. In each case, the largest value of $E_{b}$ was selected so that all waves with $k \geqslant 1$ are linearly stable. In the nonlinear calculations that follow we fix matters by taking a periodic domain of length $2 \pi$ so that the mode $k=1$ is stable or slightly unstable, depending on the value of $E_{b}$. This in turn enables us to use extensive nonlinear calculations to probe the interaction of near-wall dynamics with the electric field.

For case (a) above, an electric field strength of $E_{b}=4.5$ is found to be sufficient to completely suppress the instability. Hence we carry out a number of calculations of (41) for $0 \leqslant E_{b} \leqslant 4.5$ in order to study features beyond the linear regime. The domain is fixed to be $2 \pi$ periodic and the initial condition used is

$$
h(x, 0)=-5 \times 10^{-4} \cos x
$$

so its minimum is in the center of the computational domain and its amplitude is small enough to allow the instability to grow through its linear stage when unstable.

Figure 3 presents the dynamics of the interface as a function of time and electric field strength. The left panel depicts the interfacial maximum denoted by $h_{\max }$ and the right panel the interfacial minimum $h_{\text {min }}$. In the cases where $E_{b}<4.5$, an initial linear exponential growth occurs until the interface encounters the upper wall and then converges slowly to a state where $h_{\max }(t)$ is asymptotically close to $y=1$; at the same time, $h_{\min }(t)$ reaches a value that depends on $E_{b}$. The extracted linear growth rates are in excellent agreement with the analytically predicted values; differences do not exceed $0.01 \%$ and graphical results are omitted here for brevity. As expected, the presence of the electric field acts to delay the motion of the interface towards the wall. For example, the number of dimensionless time units required for the interfacial maximum $h_{\max }$ to reach $y=0.9$ is 

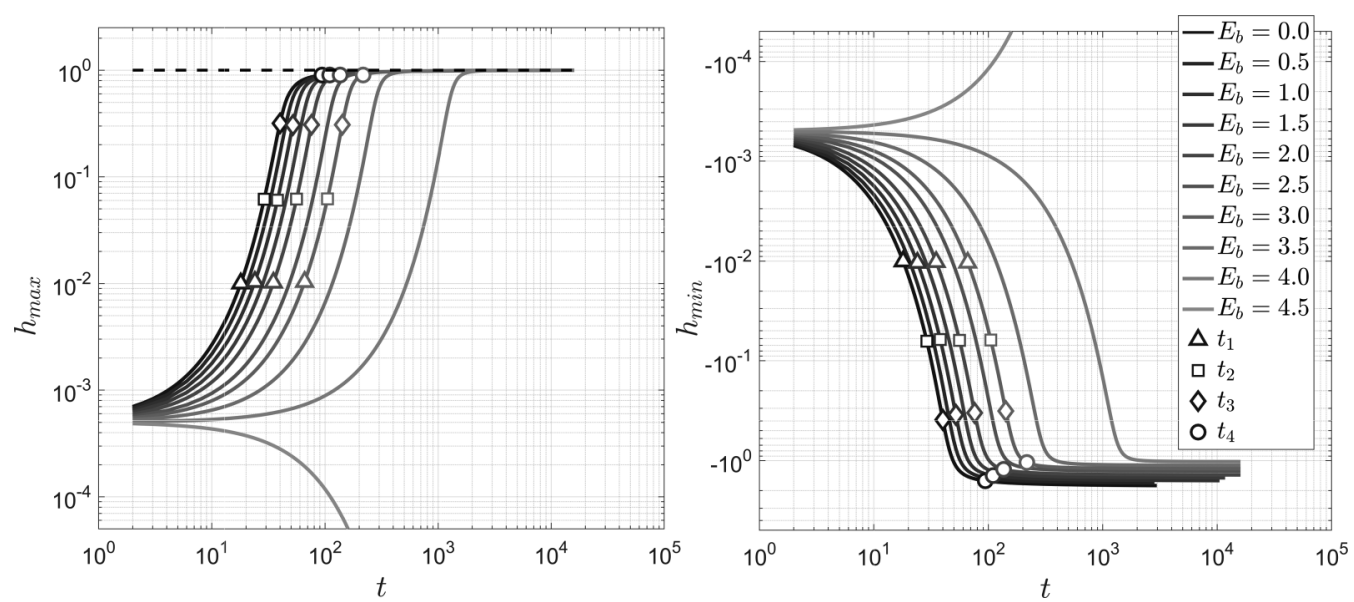

FIG. 3. Evolution of maximum $h_{\max }(t)$ and $\operatorname{minimum} h_{\min }(t)$ positions of the interface in time for $W_{e}=0.5$, $\epsilon_{p}=1.5, \epsilon_{B}=\epsilon_{T}=1.0625$, and varying $E_{b}$.

94 when $E_{b}=0.0$ and increases to 2000 when $E_{b}=4.0$, which is the final computation considered before $E_{b}$ becomes sufficiently high so as to stabilize the interface to a flat state. Several points in the figure have been marked with symbols to serve as points of comparison with subsequent direct numerical simulations. We define $t_{i}$ to denote the first time at which $h_{\max }$ exceeds a given threshold $y_{i}$, with four such values chosen as $y_{1}=10^{-2}, y_{2}=6 \times 10^{-2}, y_{3}=5 \times 10^{-1}$, and $y_{4}=9 \times 10^{-1}$; the last value is sufficiently close to the wall such that nonlinear features become prominent. We emphasize that the nonlinear draining behavior described in this confined system is in qualitative contrast with the evolution into the well-known mushroom shape of the Rayleigh-Taylor instability found in vertically unbounded domains [18]. In the present study the liquid films are not thick enough to support dripping dynamics; instead the fluid gathers into structures comprised of collars and smaller-amplitude secondary lobes separated by slowly thinning regions [5].

Our computations indicate that one of the main properties affected by the electric field is the geometry of the evolving interface as measured by aspect ratio $\chi=\left|h_{\min }(t)\right| /\left|h_{\max }(t)\right|$, for example; this serves as a measure of the distortion of the interface when compared to its initial regular cosine profile. Results corresponding to case (a) parameters are given in Fig. 4(a). In the absence of electric

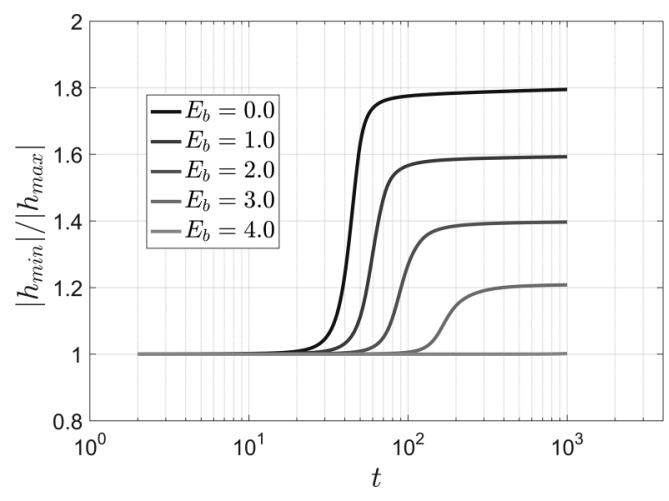

(a)

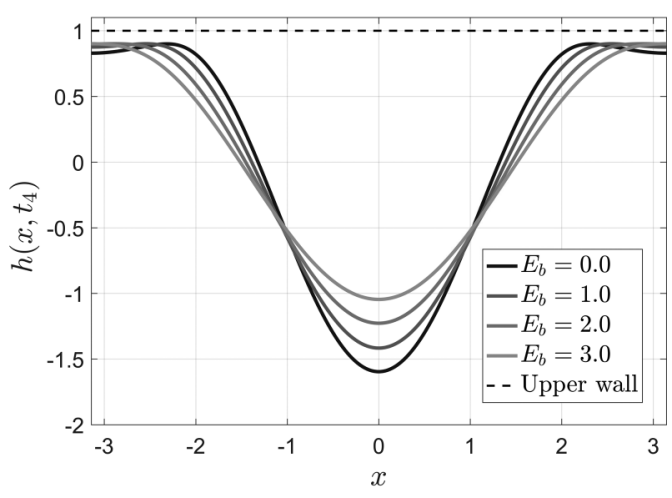

(b)

FIG. 4. Shape dynamics for $W_{e}=0.5, \epsilon_{p}=1.5$, and $\epsilon_{B}=\epsilon_{T}=1.0625$ : (a) $\left|h_{\min }(t)\right| /\left|h_{\max }(t)\right|$ and (b) interfacial shape at $h_{\max }=0.9$. 


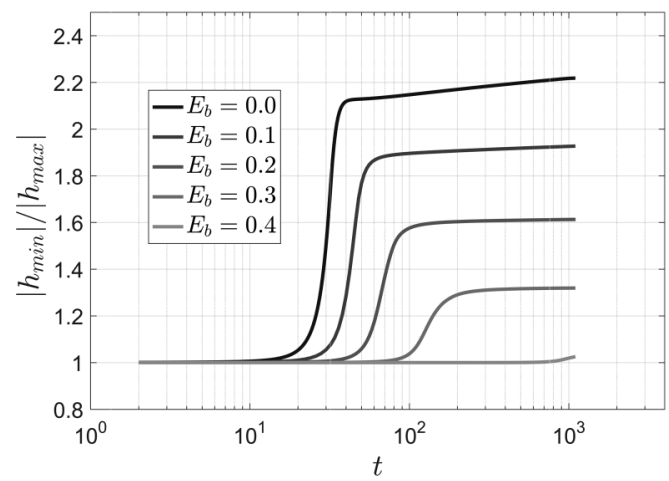

(a)

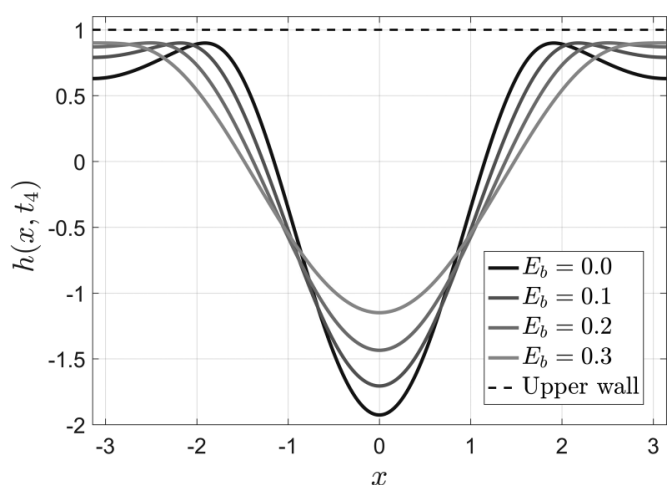

(b)

FIG. 5. Shape dynamics for $W_{e}=0.35, \epsilon_{p}=3.0$, and $\epsilon_{B}=\epsilon_{T}=1.5$ : (a) $\left|h_{\min }(t)\right| /\left|h_{\max }(t)\right|$ and (b) interfacial shape at $h_{\max }=0.9$.

effects $E_{b}=0$, the nonlinear stage starts at $t \approx 20.0$ and we find a sharp increase in $\chi$ from 1.0 to almost 1.8. This alludes to a motion of interfacial maxima inward from the sides of the domain to accommodate the extension of the minimum further down. Indeed, by observing the shapes when the maximum reaches $y=0.9$ [see Fig. 4(b)], we notice a shift of the maxima from $\pm \pi$ to approximately \pm 2.25 , with further inward migration as time progresses. The main fluid body is complemented in this case by the formation of a secondary lobe containing a (relatively) small amount of fluid. The electric field acts towards a delay in the onset of the formation of this structure, which also leads to a reduction in size of the volume of fluid within. Comparing interfacial shapes with the same $h_{\max }$ during their evolution, we find that as $E_{b}$ increases the two maxima are found closer to $\pm \pi$, and for $E_{b}=4.0$ the initial cosine shape is actually retained until the end of the computation. Referring to Fig. 4(a), $\left|h_{\min }(t)\right| /\left|h_{\max }(t)\right|$ decreases steadily as a function of $E_{b}$ down to unity, which indicates regularization to a sinusoidal shape of the interface as the electric field contribution is increased.

A similar trend is found for the second choice of parameters, case (b) above, with the interfacial extrema dynamics shown in Figs. 5(a) and 5(b). In this case the surface tension effects are smaller and the nonlinearity in the interface becomes more pronounced, with $\chi$ reaching 2.2 when no electric field is present. The instability threshold for this set of parameters is $E_{b} \approx 0.416$ and $\chi \rightarrow 1$ at all values of $E_{b}$ above this.

The nonlinear computations extend to $O\left(10^{3}-10^{4}\right)$ time units, which enables further insight into the long-term dynamics of the system. Quantifying the regularization of the interfacial shapes as a function of the electric field strength is best illustrated by considering the aspect ratios $\chi$ of the saturated profiles, which are extracted from the final time of each computation. At this stage all maxima are within $10^{-2}$ of the wall and going beyond this minimum thickness requires prohibitively long computations. Figure 6 illustrates the ratio $\chi=\left|h_{\min }\right| /\left|h_{\max }\right|$ as a function of $E_{b}$ for both example cases discussed up to this point. The same salient feature is observed in both scenarios, namely, that up to the point at which $E_{b}$ is sufficiently large to stabilize the flow, the aspect ratios vary linearly with $E_{b}$; the final profiles used to extract these data start from distorted nonlinear profiles in the absence of a field and end up with $\chi=1$, which corresponds to a regular cosine shape when $E_{b}$ is large enough to be close to its complete stabilization value. The negative slope varies depending on the remaining parameters $W_{e}, \epsilon_{p}, \epsilon_{B}$, and $\epsilon_{T}$; however, once a critical value of $E_{b}$ is reached (close to the linear stability threshold), $\chi$ becomes unity and remains so beyond the threshold.

The modification in the interfacial shape characteristics may also be understood in view of the amount of liquid contained in the primary structures of the profile. When the electric field is absent we find a large-collar and a small-amplitude lobe coexisting after sufficiently long times. The lobes are centered symmetrically at $x= \pm \pi$ and extend to $x= \pm a$ where $a<\pi$; the interface has local maxima at $x= \pm a$. To facilitate comparisons between different computations, we consider the 


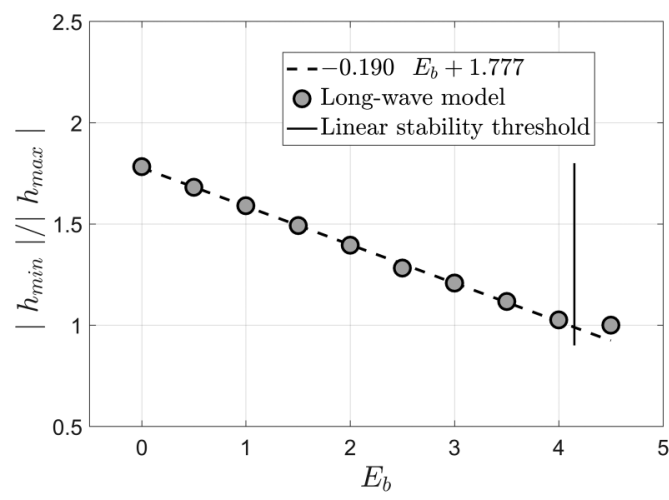

(a)

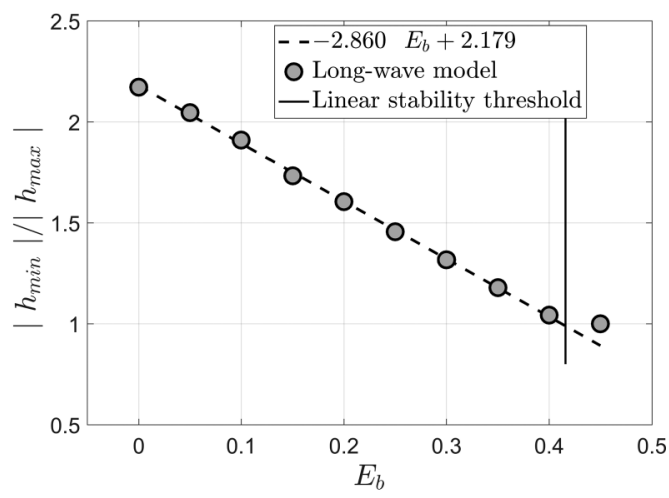

(b)

FIG. 6. Aspect ratio as a function of the electric field strength for the saturated profiles: (a) $W_{e}=0.5$, $\epsilon_{p}=1.5$, and $\epsilon_{B}=\epsilon_{T}=1.0625$ and (b) $W_{e}=0.35, \epsilon_{p}=3.0$, and $\epsilon_{B}=\epsilon_{T}=1.5$.

interfacial profiles at final times when $1-h_{\max }<0.01$, i.e., the interface is at a distance of 0.01 from the wall. Using this condition, we find that when $E_{b}=0$ the lobe edges are at the coordinates \pm 2.24 . The main collar formed between these points contains $98.77 \%$ of the total liquid in the film, with the remaining small quantity shared by the secondary lobes.

As the electric field strength is increased the lobe edge coordinates move towards the domain edges. This is illustrated in Fig. 7(a) for case (a) and up to the value $E_{b}=4.0$ when the stabilizing action of the nonzero voltage prevents lobe formation. In general, an increase in $E_{b}$ reduces the volume of the secondary lobes; these disappear completely and all the liquid is in the main collar beyond a certain threshold value of $E_{b}$. The second parameter study for case (b) reveals a similar behavior, with anticipated quantitative differences. Figure $7(\mathrm{~b})$ indicates a more pronounced displacement of the maxima, with the secondary lobes being more prominent for smaller values of $E_{b}$. Less than $94.24 \%$ of the liquid lies inside the main collar when no voltage potential difference is applied, with the stronger distortion owing to the reduced contribution of surface tension. When $E_{b} \geqslant 0.4$, the secondary lobe disappears with the liquid draining inside a single collar occupying the entire horizontal extent of the domain.

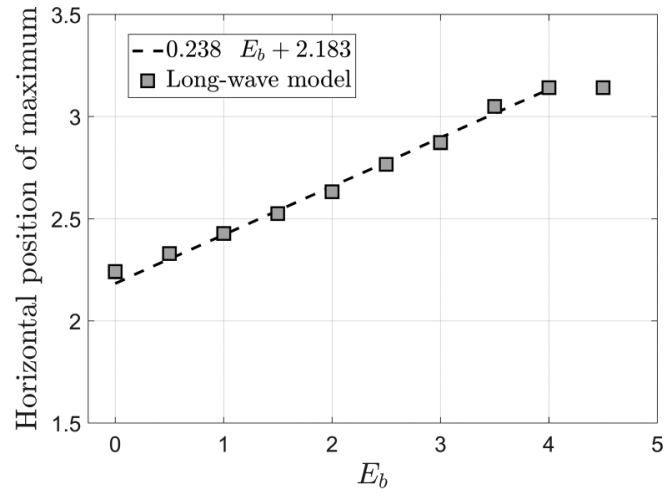

(a)

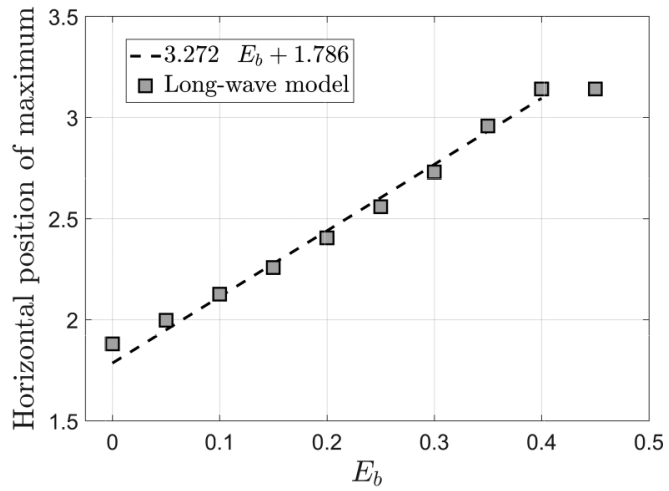

(b)

FIG. 7. Interfacial maximum horizontal coordinate considered in absolute value as the distance from the origin at $x=0$ in a domain of size $[-\pi, \pi]$ for (a) $W_{e}=0.5, \epsilon_{p}=1.5$, and $\epsilon_{B}=\epsilon_{T}=1.0625$ and (b) $W_{e}=0.35, \epsilon_{p}=3.0$, and $\epsilon_{B}=\epsilon_{T}=1.5$. All data have been extracted at the time step at which the interfacial maxima first approach the upper wall to within a distance of less than 0.01 . 


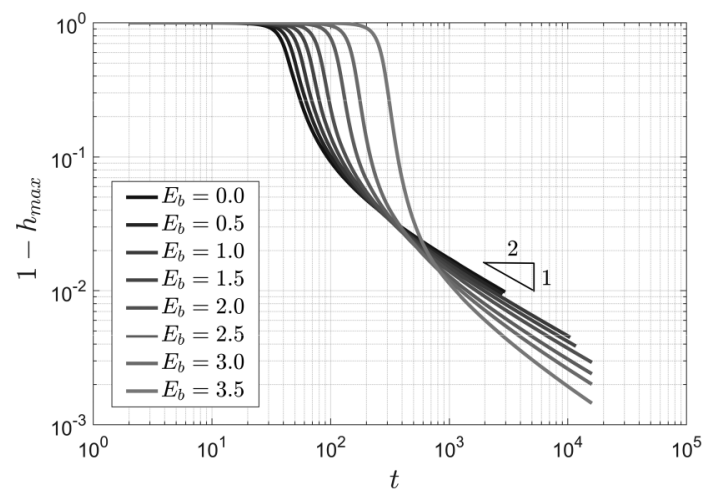

(a)

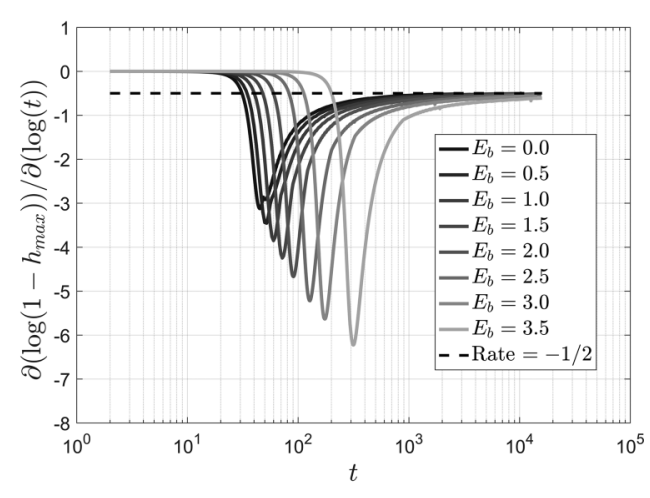

(b)

FIG. 8. Asymptotic behavior of the interfacial maximum $h_{\max }$ as the interface approaches the wall for $W_{e}=0.5, \epsilon_{p}=1.5, \epsilon_{B}=\epsilon_{T}=1.0625$, and a selection of electric field strength values $E_{b}$ : (a) distance from wall of $h_{\max }(t)$ and (b) draining rate.

Finally, we consider the draining rate of the fluid, which is the rate at which the interfacial maximum approaches the wall. In accord with the similarity solutions of [21] and [4] and also in good agreement with the more recent exploration of [5], we find this rate to be $\left(1-h_{\max }\right) \propto t^{-1 / 2}$; these results are presented using a log-log scale in Fig. 8 for the first set of parameters, case (a). The second test case (b) follows a similar pattern. While the number of time units required to reach this rate increases with $E_{b}$, we find that for all values of $E_{b}$ tested where instability is supported, the algebraic thinning rate follows the $1 / 2$ power law, thus suggesting that the electric field does not enter into the dominant asymptotic balances.

\section{DIRECT NUMERICAL SIMULATIONS}

To complement our asymptotic study and to further explore the dynamics in the fully nonlinear nonslender regime, we carry out direct numerical simulations of the problem using the volumeof-fluid software GERRIS [22,23]. The second-order accuracy in both time and space of the finitevolume formulation, coupled with computational capabilities such as adaptive mesh refinement and parallelization features, contributes to a highly efficient numerical methodology. We refer the interested reader to our previous work [18] for an outline of relevant numerical aspects in the context of a related electrohydrodynamical problem.

\section{A. Methodology}

A sketch of the problem has been given in Fig. 1. In the direct numerical simulations the action of the lower and upper solids is retained and these are modeled as highly dense and viscous fluids. In addition, the associated surface tension between fluids 0 and 1 , as well as 2 and 3 , is taken to be large. The bottom and top layers emulate the effects of solids playing a passive role in the fluid dynamical solution, with only the electric problem being of interest there. The direct numerical simulation (DNS) setup is two dimensional and all fluids are considered to be incompressible, immiscible, and viscous. They are also considered to be perfect dielectrics.

A different nondimensionalization procedure is followed for the DNS in order to improve the stability of the discretization scheme involved. In what follows stars are used to denote dimensional quantities. The governing equations are the Navier-Stokes equations (1) and the Laplace equation for the voltage potentials (3), where it is understood that these now hold in each domain $0,1,2$, and 3. Electric contributions appear in the form of jumps at the interface in the Maxwell stresses in the 
appropriate terms of the normal stress balance (see [18] for details on how these are incorporated as source terms in the momentum equations).

We use the horizontal length of the channel $L_{D}^{*}$ as reference length and $U^{*}=\sqrt{g^{*} L_{D}^{*}}$ as reference velocity. With the exception of permittivity, the physical properties of fluid 2 are used as reference and pressures are scaled by $\rho_{2}^{*} U^{* 2}$. The permittivities are scaled with respect to the values in fluid 1. There are four main dimensionless groups arising:

$$
\tilde{g}=\frac{g^{*} L_{D}^{*}}{U^{* 2}} \equiv 1, \quad \tilde{\mu}=\frac{\mu_{2}^{*}}{\rho_{2}^{*} \sqrt{g^{*} L_{D}^{*}} L_{D}^{*}}, \quad \sigma=\frac{\sigma_{12}^{*}}{\rho_{2}^{*} g^{*} L_{D}^{* 2}}, \quad E_{b}=\frac{\epsilon_{1}^{*} V_{0}^{* 2}}{\rho_{2}^{*} g^{*} L_{D}^{* 3}} \equiv 1 .
$$

The quantity $\tilde{g}$ represents an inverse-squared Froude number and setting it to 1 allows us to recover a suitable reference velocity; $\tilde{\mu}$ is an inverse Reynolds number acting as dimensionless viscosity; an inverse Weber number $\sigma$ acts as dimensionless surface tension. The dimensionless electric field group $E_{b}$ is set to 1 and provides a reference voltage in the system given by $V_{0}^{*}=\sqrt{\rho_{2}^{*} g^{*} L_{D}^{* 3} / \epsilon_{1}^{*}}$. The quantity $\bar{V}=\bar{V}^{*} / V_{0}^{*}$ (note that the electric field is $E^{*}=\bar{V}^{*} / L_{D}^{*}$ ) measures the magnitude of the applied voltage potential difference. For notational convenience we introduce the following physical property ratios:

$$
\begin{gathered}
r_{0}=\rho_{2}^{*} / \rho_{0}^{*}, \quad r_{1}=\rho_{2}^{*} / \rho_{1}^{*}, \quad r_{3}=\rho_{2}^{*} / \rho_{3}^{*}, \quad m_{0}=\mu_{0}^{*} / \mu_{2}^{*}, \quad m_{1}=\mu_{1}^{*} / \mu_{2}^{*}, \quad m_{3}=\mu_{3}^{*} / \mu_{2}^{*}, \\
\epsilon_{B}=\epsilon_{0}^{*} / \epsilon_{1}^{*}, \quad \epsilon_{T}=\epsilon_{3}^{*} / \epsilon_{1}^{*}, \quad \epsilon_{p}=\epsilon_{2}^{*} / \epsilon_{1}^{*}, \quad s_{10}=\left(\sigma_{10}^{*} / \sigma_{12}^{*}\right) \sigma, \quad s_{32}=\left(\sigma_{32}^{*} / \sigma_{12}^{*}\right) \sigma
\end{gathered}
$$

The quantities $\sigma_{10}^{*}, \sigma_{12}^{*}$, and $\sigma_{32}^{*}$ denote the surface tension coefficients between the fluids in regions 1 and 0,1 and 2 , and 3 and 2 , respectively. The dimensionless domain has unit horizontal extent and lies in $-1 / 2<x<1 / 2$. For the hydrodynamics at the end points $x= \pm 1 / 2$ we impose impermeability $u( \pm 1 / 2, y, t)=0$ and free slip $v_{y}( \pm 1 / 2, y, t)=0$. A voltage potential difference $\bar{V}$ is maintained by prescribing a nonzero voltage $\left.\phi\right|_{x=-1 / 2}=\bar{V}$ on the left boundary, with $\left.\phi\right|_{x=+1 / 2}=0$

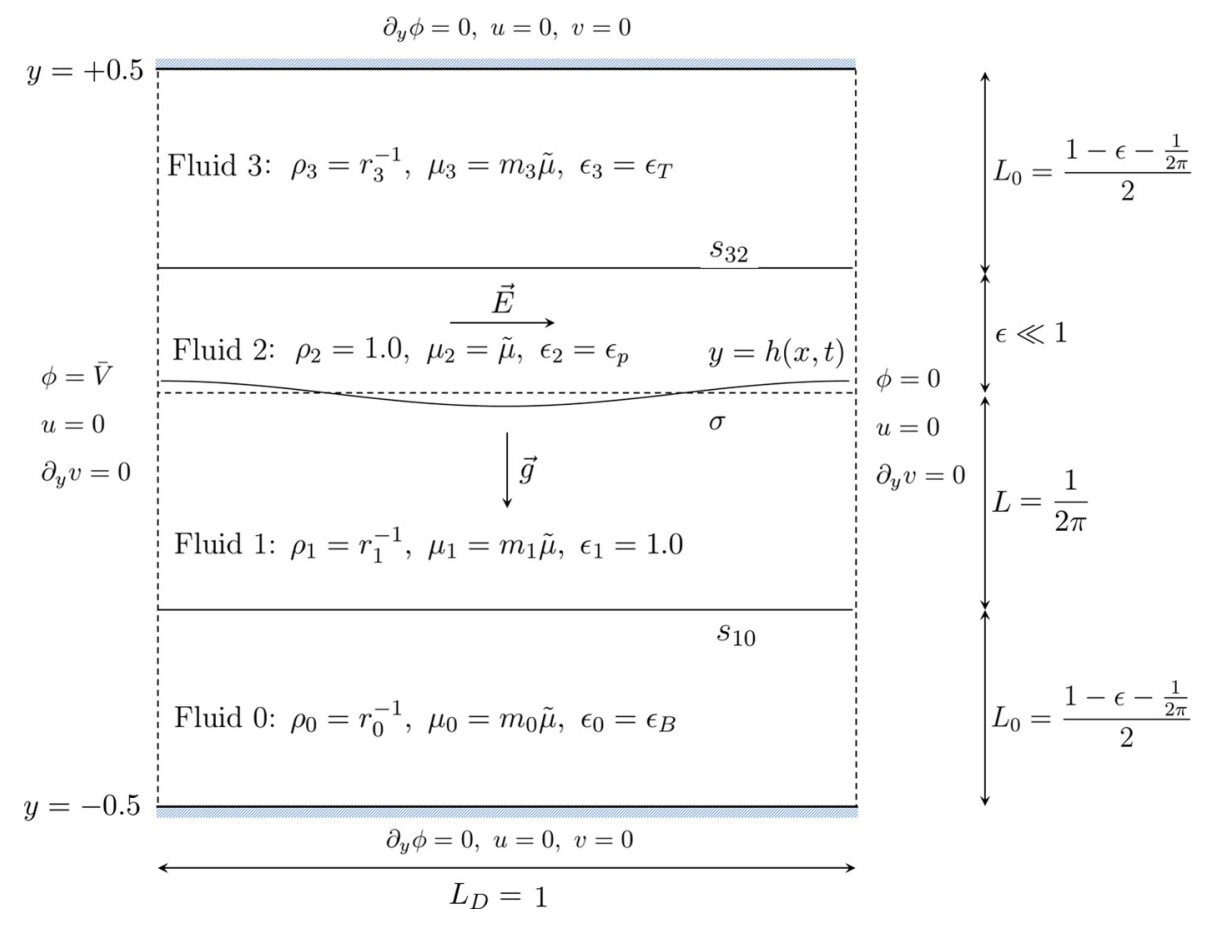

FIG. 9. Schematic of the computational domain utilized in the direct numerical simulations. 
on the right. The dimensionless liquid layer region 2 has mean thickness $\epsilon$, while region 1 lying below it has dimensionless mean thickness $\frac{1}{2 \pi}$. The bounding slab regions 0 and 3 are taken to have a finite vertical thickness of dimensionless size $L_{0}=\frac{1}{2}\left(1-\epsilon-\frac{1}{2 \pi}\right)$, rather than the semiinfinite extent employed in the analysis. More specifically, in their undisturbed states, region 0 occupies $-\frac{1}{2}<y<-\frac{1}{2}+L_{0}$, region 1 occupies $-\frac{1}{2}+L_{0}<y<-\frac{1}{2}+L_{0}+\frac{1}{2 \pi}=\frac{1}{4 \pi}-\frac{\epsilon}{2}$, region 2 occupies $\frac{1}{4 \pi}-\frac{\epsilon}{2}<y<\frac{1}{4 \pi}+\frac{\epsilon}{2}$, and region 3 occupies $\frac{1}{4 \pi}+\frac{\epsilon}{2}<y<\frac{1}{2}$. At the outer walls $y=$ $-\frac{1}{2}$ and $y=\frac{1}{2}$ we impose no-slip conditions $u=v=0$ and zero vertical electric field components $\partial_{y} \phi_{0}(x,-1 / 2, t)=0$ and $\partial_{y} \phi_{3}(x, 1 / 2, t)=0$. Note that while in the asymptotic model regions 0 and 3 are considered to be semi-infinite, we found that imposing a wall at a finite distance $L_{0}$ away has inconsequential effects on the flow; furthermore, numerical experiments showed that $L_{0}=O\left(10^{-1}\right)$ is sufficient. For completeness, the schematic of the domain utilized in the DNS is included in Fig. 9.

A sinusoidal initial perturbation of the fluid interface $y=h(x, t)$ is imposed

$$
h(x, 0)=-A \cos (2 \pi q x),
$$

where $A$ is the amplitude and the integer $q \geqslant 1$ enables variation in the wave number. In all the runs presented here we have taken $q=1$, which corresponds to a wave as long as the domain size. In addition, we select small $A=O\left(10^{-5}\right)$ so that the interfacial minimum is located at $x=0$ when $q$ is odd. In the following section we present results that compare the interfacial dynamics obtained in the long-wave asymptotic framework with the direct numerical simulations.

\section{B. Comparison of DNS with thin-film model results}

Recall that the solutions of the evolution equation (41) depend on five dimensionless physical parameters: the surface tension parameter $W_{e}$, the electric field strength $E_{b}$, and the permittivity ratios $\epsilon_{B}, \epsilon_{T}$, and $\epsilon_{p}$. The thin liquid layer assumption, along with the passive nature of the fluid in region 1, enabled analytical progress and derivation of a tractable partial differential equation (PDE) that retains desired physical effects. By contrast, the direct numerical simulations employing the volume-of-fluid methodology introduce additional complexity. First, the small liquid layer height $\epsilon \ll 1$ was scaled out of the analysis; however, a suitable choice is required when modeling the full two-dimensional computational domain. It is anticipated that if $\epsilon$ in the DNS is sufficiently small, then the qualitative impact on the solution will be limited. However, quantitative differences, as well as time-scale discrepancies in the evolution of the flow, will arise when considering thresholds for instability windows. A second element absorbed within the theoretical derivation is the $O(1)$ Reynolds number, which is embedded in the time derivative of the interface. This implies that variation of the inverse Reynolds number $\tilde{\mu}$ defined in Eq. (44) affects the time scale and growth rate of the instability but only has a marginal effect on the interfacial shape and other nonlinear features. Finally, concrete choices must be made for the density ratios $r_{0}, r_{1}$, and $r_{3}$ and the viscosity ratios $m_{0}, m_{1}$, and $m_{3}$ so that the fluid system is Rayleigh-Taylor unstable and the contrast in physical quantities is sufficiently strong to reflect the idealized configuration of an upper liquid layer with a passive fluid underneath.

The challenges described above render a one-to-one correspondence with the asymptotic solutions very difficult to attain. Furthermore, the DNS are made even more expensive due to the slow time scale of the flow for small $\epsilon$ and as wall touching takes place. Resolving such layer thinning dynamics for the model equation required $O\left(10^{3}-10^{4}\right)$ time units (see Fig. 8). Noting the $\epsilon^{-3}$ rescaling in the time variable [see Eq. (26)] and the smallness of $\epsilon$, it is anticipated that the number of time units needed to observe the same features in the DNS will increase prohibitively by a factor of at least $10^{3}$ for $\epsilon$ smaller than 0.1 . Two simplifications that arose from extensive numerical experimentation were introduced to surmount these difficulties. The value for $\epsilon$ has been set to $0.2 L=0.2 / 2 \pi$, which was found to be sufficiently small to reproduce the findings of the model. Second, the Reynolds number used in the computations has been increased by a factor of approximately 3 , so when recast into the nondimensionalization (44), its inverse becomes $\tilde{\mu}=0.02$ instead of 0.0635 , which would directly 

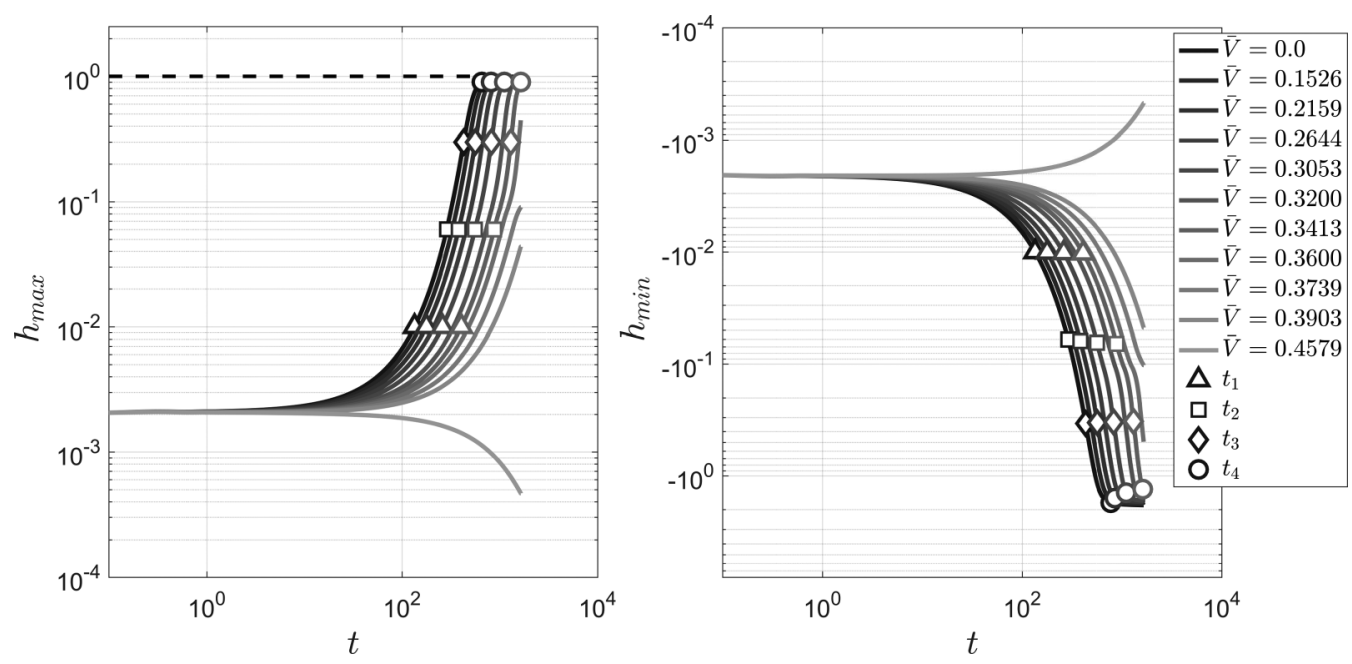

FIG. 10. Evolution of maximum $h_{\max }(t)$ and minimum $h_{\min }(t)$ positions of the interface in time for $\sigma=$ $0.012665, \epsilon_{p}=1.5, \epsilon_{B}=\epsilon_{T}=1.0625$, and varying $\bar{V}$. The remaining physical parameters for the DNS are $\tilde{\mu}=0.02, s_{10}=s_{32}=39.47, r_{0}=1.0, r_{1}=80.0, r_{3}=10.0, m_{0}=5.0, m_{1}=1.0$, and $m_{3}=1.0$.

coincide with $\mathrm{Re}=1$ used in the model. These parameter choices accelerate the computations significantly and provide results from direct numerical simulations that can be compared with the asymptotic model solutions. Adaptive grid refinement is used, which is set to increase resolution in the vicinity of the interface and the thin liquid layer. Adaptivity allows for coarser grids away from the thin layer, but a large number of degrees of freedom emerges still. For example, prescribing the highest level of refinement around the interface to be 256 cells, adaptivity yields approximately $5 \times 10^{3}$ degrees of freedom; this is an order of magnitude less than what it would be over a uniform grid at the resolution of the interface. Nonetheless, to capture the dynamics of the interface as it gets close to the wall we require at least 1000 CPU hours for each run.

For the first test case we take a surface tension coefficient $\sigma=0.012665$ and permittivity ratios $\epsilon_{p}=1.5$ and $\epsilon_{B}=\epsilon_{T}=1.0625$, all being equivalent to the values used in the study of the long-wave model in Sec. III. The imposed voltage potential difference $\bar{V}$ varies from 0.0 to 0.46 , with the threshold for instability for a wave number of $2 \pi$ found to be of $\bar{V} \approx 0.42$. A considerably higher surface tension coefficient is used for the lower and upper interfaces between layers 0 and 1 and between layers 2 and 3, respectively, with $s_{10}=s_{32}=39.47$. As intended, the hydrodynamic interaction between the lower two layers is very limited, with the stably stratified system, strong surface tension, and flat interface all contributing to replicating the boundary between a liquid and a solid in which the electric terms are the only non-negligible contribution. The second test case is characterized by $\sigma=0.008865, \epsilon_{p}=3.0, \epsilon_{B}=\epsilon_{T}=1.5$, and $\bar{V}$ varying from 0.0 to 0.235 , with $s_{10}=s_{32}=56.5$. The remaining physical parameters pertaining to the direct numerical simulations in both studies are density ratios $r_{0}=1.0, r_{1}=80.0$, and $r_{3}=10.0$, viscosity ratios $m_{0}=5.0$, $m_{1}=1.0$, and $m_{3}=1.0$, and inverse Reynolds number $\tilde{\mu}=0.02$.

The time evolution of the scaled interfacial maximum and minimum are depicted in Fig. 10, to be compared to the analogous Fig. 3 in the asymptotic study. To enable comparisons with the model computations, the interfacial position for the DNS is written as $h(x, t)=\frac{1}{4 \pi}-\frac{\epsilon}{2}+\epsilon \tilde{h}(x, t)$ and hence the scaled interface is $\tilde{h}(x, t)=\left[h(x, t)-\frac{1}{4 \pi}+\frac{\epsilon}{2}\right] / \epsilon$, where $\epsilon=0.2 / 2 \pi$ in the simulations; it follows that $-5<\tilde{h}<1$, with the lower bound due to the upper limit of fluid region 0 at $y=-0.5+L_{0}$. The results of Fig. 10 also show that an increase of $\bar{V}$ reduces the instability and complete stabilization is achieved above a critical value of $\bar{V} \approx 0.42$. Interestingly, due to the imposition of a combination of Neumann and Dirichlet lateral boundary conditions for the fluid 

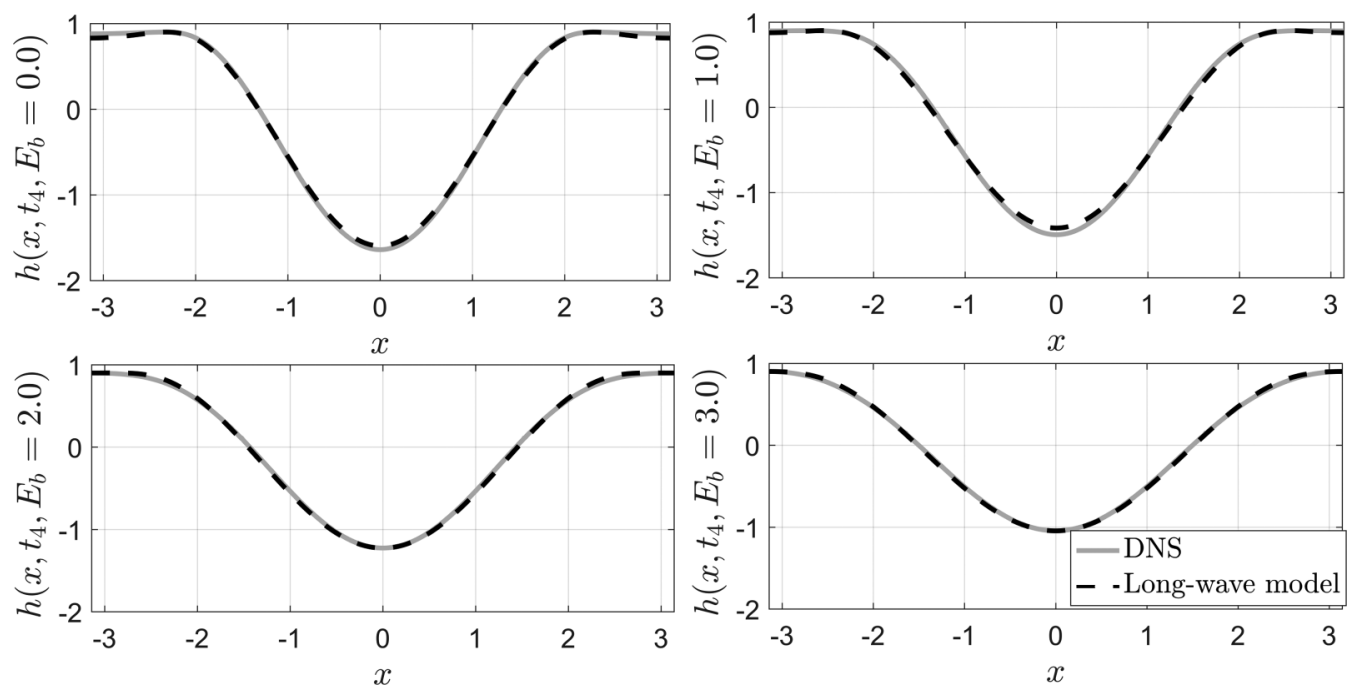

FIG. 11. Interfacial shape comparison as $h_{\max }=0.9$ for increasing values of $E_{b}$ (dots labeled $t_{4}$ in Fig. 3 for the long-wave model) and $\bar{V}$ (dots labeled $t_{4}$ in Fig. 10 for the direct numerical simulations) for the first choice of parameters $\sigma=0.012665, \epsilon_{p}=1.5$, and $\epsilon_{B}=\epsilon_{T}=1.0625$. In addition, $E_{b}=0.0$ (top left), $E_{b}=1.0$ (top right), $E_{b}=2.0$ (bottom left), and $E_{b}=3.0$ (bottom right).

velocities (as opposed to periodic boundary conditions in the long-wave model), the flow eventually becomes unstable since subharmonic instabilities are now supported, i.e., even though the $k=1$ mode is stable, the $k=1 / 2$ mode is unstable and is allowed by the boundary conditions. Numerically we can see subharmonic instabilities appearing even when the interfacial perturbation drops below $10^{-8}$ due to the stabilization of the $k=1$ mode introduced by the initial condition.

The shapes of the interface obtained from the DNS are compared to their counterparts from the long-wave model in Figs. 11 and 12 for the two different cases investigated. The four panels
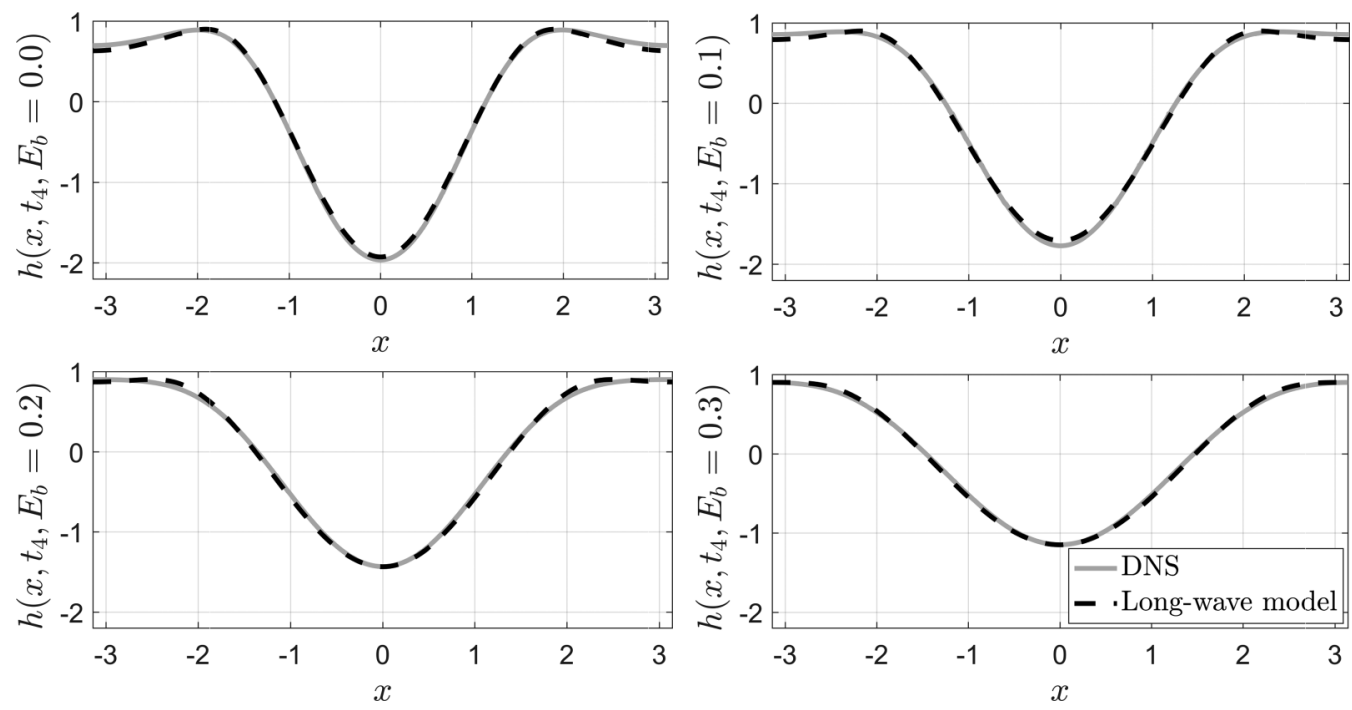

FIG. 12. Interfacial shape comparison as $h_{\max }=0.9$ for increasing values of $E_{b}$ and $\bar{V}$ for the second choice of parameters $\sigma=0.008865, \epsilon_{p}=3.0$, and $\epsilon_{B}=\epsilon_{T}=1.5$. In addition, $E_{b}=0.0$ (top left), $E_{b}=0.1$ (top right), $E_{b}=0.2$ (bottom left), $E_{b}=0.3$ (bottom right). 


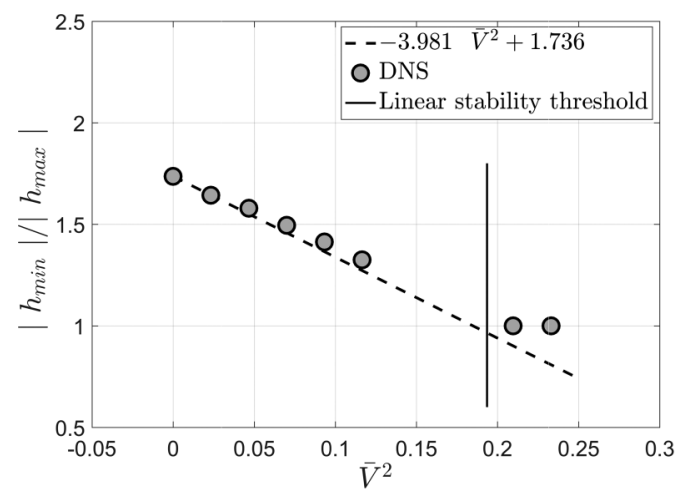

(a)

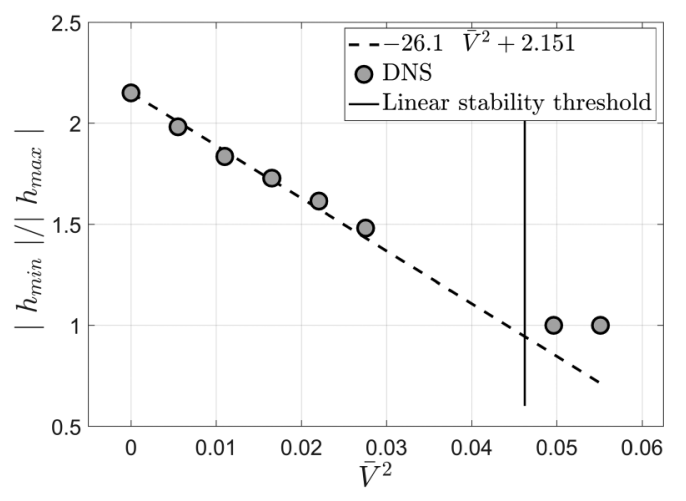

(b)

FIG. 13. Aspect ratio as a function of the electric field strength for the saturated profiles based on results originating from the direct numerical simulations: (a) $\sigma=0.012655, \epsilon_{p}=1.5$, and $\epsilon_{B}=\epsilon_{T}=1.0625$ and (b) $\sigma=0.008865, \epsilon_{p}=3.0$, and $\epsilon_{B}=\epsilon_{T}=1.5$.

show the superimposed interfaces when $h_{\max }$ first exceeds 0.9, indicated by circles in Figs. 3 and 10. Excellent agreement is found between long-wave and DNS results, with the nonlinearity in the shape being regularized to a cosine shape as the electric field is increased. More pronounced features are observed in the second case due to a smaller value of $\sigma$. For values of the field near the stabilization threshold, our results indicate that nonlinear distortion is reduced and the shapes grow towards the wall retaining their initially sinusoidal profiles.

The dependence between the interfacial shape aspect ratio $\chi=\left|h_{\min }\right| /\left|h_{\max }\right|$ as a function of the electric field strength is summarized in Fig. 13. Given the linear dependence of $\chi$ on $E_{b}$ once saturation is reached in the long-wave model (see Fig. 6), we anticipate a similar behavior in the DNS. Noting that $\bar{V} \sim \sqrt{E_{b}}$ with $E_{b}$ defined by (10), we expect a linear variation of $\chi$ with $\bar{V}^{2}$. This is indeed the case in the results of Fig. 13; the slope and intercept vary as a function of the numerous parameters in the flow. The slopes of the two data sets do not match exactly as we do not anticipate a one-to-one correspondence of the results at finite $\epsilon$. We also note that it is very difficult to obtain data for moderate to large values of $\bar{V}$ where the flow is very close to the stability threshold; due to very slow development of the instability, this requires $O\left(10^{4}\right)$ CPU hours for each computation. In such cases the advantages of employing the asymptotic model become evident. Making $\epsilon$ bigger reduces computational times but can also violate the assumptions underlying the long-wave model that we are trying to compare with. Given the excellent agreement between the model and the DNS, we consider next in Sec. IV C the range of validity of the model by carrying out detailed DNS studies as $\epsilon$ increases. We note (results not shown) that when we increase the vertical extent of the lower side of the domain and remove the lower fluid from the system, dripping can occur should $\epsilon$ exceed a threshold value for a given $\bar{V}$.

\section{Using DNS to test the validity of the long-wave equations}

Even though the evolution equation (41) is valid for $\epsilon \ll 1$, the numerical simulations of Sec. IV B have shown that agreement is excellent for $\epsilon=0.2 / 2 \pi$. In what follows we examine numerically the solution dependence on the liquid film height and characterize the dynamics at progressively larger values of $\epsilon$ in order to evaluate the validity of the model (41). In order to reduce the number of parameters we concentrate on the nonelectrified case $\bar{V}=0$.

We take parameters similar to those of the first case described in Sec. IV B, with density ratios $r_{0}=$ 1.0, $r_{1}=80.0$, and $r_{3}=10.0$, viscosity ratios $m_{0}=5.0$ and $m_{1}=m_{3}=1.0$, and surface tension coefficient $\sigma=0.012665$, while $s_{10}=s_{32}=39.47$ (permittivity ratios are no longer relevant). In 

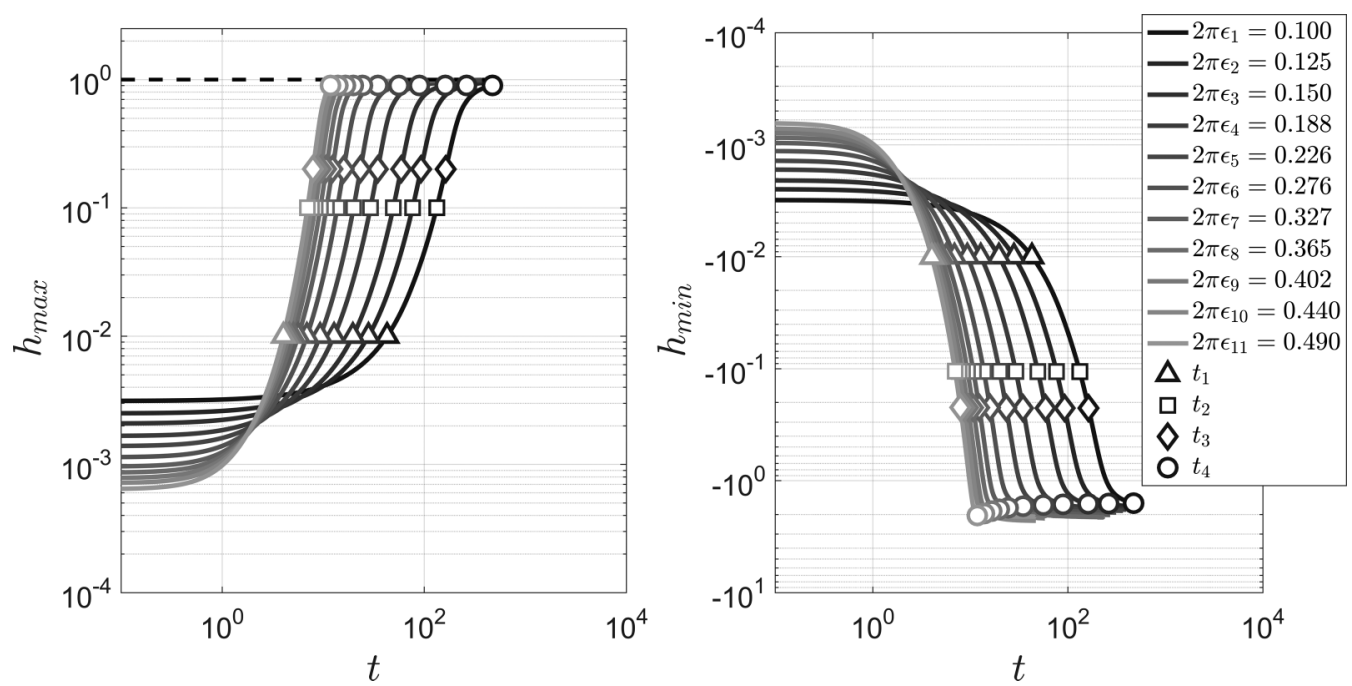

FIG. 14. Evolution in time of the scaled maximum $h_{\max }(t)$ and minimum $h_{\min }(t)$ positions of the interface as the liquid film height $\epsilon$ is increased.

order to promote a shorter time scale of the dynamics for the smaller values of $\epsilon$, the dimensionless viscosity is reduced to $\tilde{\mu}=0.001$.

Figure 14 presents results for $\epsilon$ varying between $0.1 / 2 \pi$ and $0.49 / 2 \pi$. Here we plot the interfacial extrema $h_{\max }$ and $h_{\min }$ for all test cases, normalizing each by the appropriate value of $2 \pi \epsilon_{i}, i=$ $1, \ldots, 11$; as previously, this normalization implies that the interface touches the upper wall when $h_{\max } \rightarrow 1$. In addition, the initial amplitude is $A=5 \times 10^{-3}$ in all test cases and so its normalized value varies appropriately. We find that larger values of $\epsilon$ promote a faster growth of the instability, while the added fluid mass also affects interfacial shapes sufficiently close to the upper wall. More specifically, by plotting interfacial shapes as they first reach the maximum value of $y=0.9$ (circles at $t_{4}$ in Fig. 14), we find that the increase in fluid mass pushes the maxima further inward in the domain, supporting a stronger deformation of the interface at its minimum point where $x=0.0$ [see Fig. 15(a)]. This resembles the effect obtained when reducing the surface tension coefficient in the comparison between the two different cases analyzed previously. Quantifying the shape aspect ratio

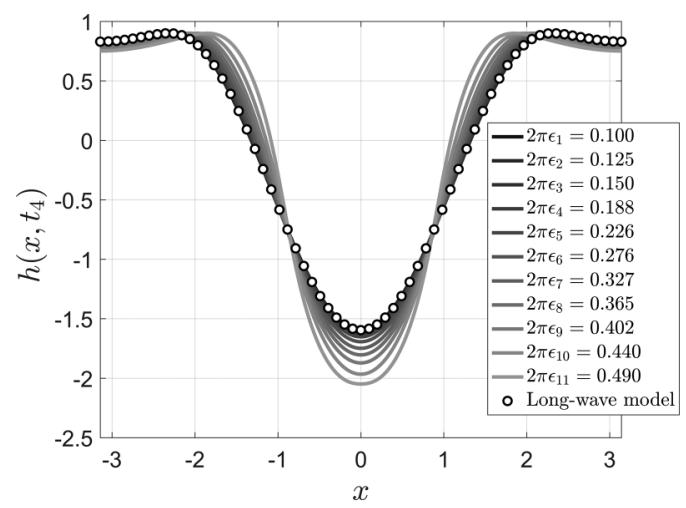

(a)

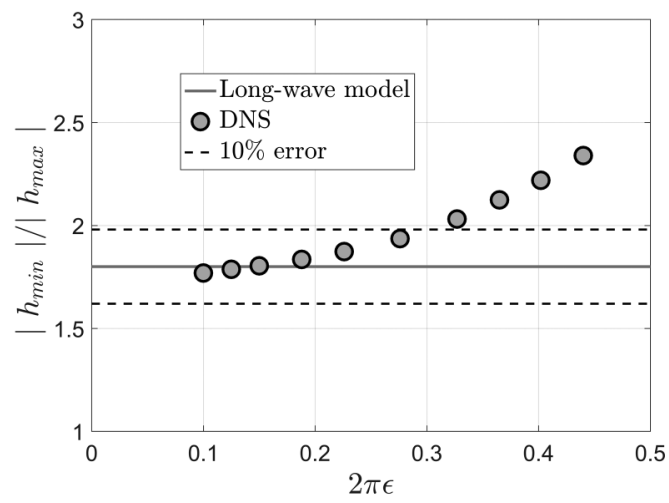

(b)

FIG. 15. Interfacial shape properties as a function of varying liquid film height $\epsilon$ : (a) scaled interfacial shapes as $h_{\max }=0.9$ and (b) aspect ratio of scaled saturated shapes. 

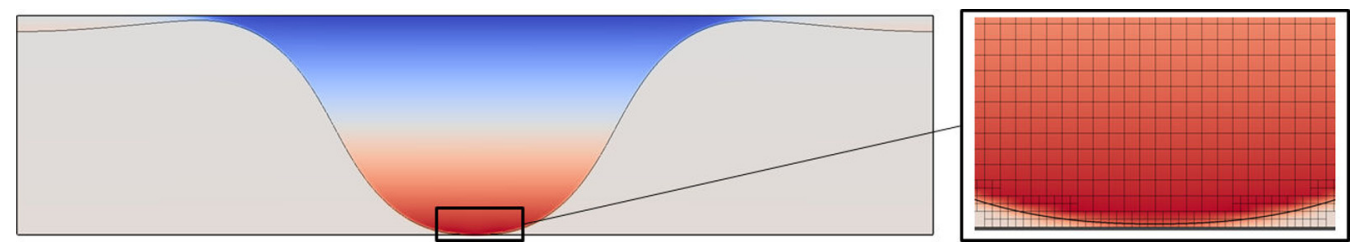

FIG. 16. Pressure field for a large $\epsilon=0.49 / 2 \pi$ case with a close-up of the region where the active fluid interface approaches the lower fluid layer and a thin region (cushion) is created between them. Beyond this stage the fluid simply extends laterally.

$\chi=\left|h_{\min }\right| /\left|h_{\max }\right|$ once the shapes have saturated shows a significant increase in the nonlinearity with $\chi$ changing from 1.78 for the smallest value of $\epsilon=0.1 / 2 \pi$ to almost 2.4 as $\epsilon$ reaches values closer to $0.5 / 2 \pi$ [see Fig. 15(b)]. Values of $2 \pi \epsilon \leqslant 0.25$ are estimated to be in good agreement (errors of less than 5\%) with the asymptotic model based on this property.

For sufficiently thick fluid layers characterized by $\epsilon>0.45$, approximately, the interface reaches the lower surface, forms a thin film there, and drains slowly (see Fig. 16). The adaptive mesh refinement illustrated in the figure ensures that such dynamics is computed accurately even as the thickness of this intermediate layer becomes very small. At this stage the upper liquid layer effectively rests on the lower surface; further increases in $\epsilon$ lead to the lateral spreading of the upper liquid on top of this (slightly compliant) surface. We note that as a result of the modeling, a small level of deformation of the lower interface is present. Further increases in the viscosity, density, and surface tension coefficient in the lower passive layer would alleviate this artifact; however, the deformation is found to be sufficiently small to have a negligible effect on the overall dynamics of the system.

The results presented indicate that the asymptotic model captures the main features of the flow well even for relatively large values of $\epsilon$. Qualitatively there are only minor modifications in the flow even until the lower surface is reached, while quantitatively we find good agreement between the results based on the long-wave evolution equations and the DNS for $2 \pi \epsilon \leqslant 0.25$.

The results thus far consider systems with relatively strong surface tension that are confined from below and hence pendant drop formation, elongation, and subsequent dripping have not been observed. We have investigated such phenomena (details omitted here for brevity) by removing the lower fluid 0 and extending region 1 vertically so that bottom wall effects become negligible; all other parameters described earlier in this section are kept fixed. We find that for $\epsilon \leqslant 0.4$, the results coincide with the confined case. When $\epsilon \approx 0.45$, minor differences emerge as the liquid layer interacts with the lower interface when the latter is present. As $\epsilon$ exceeds a value of approximately 0.5 the formation of a pendant drop and eventual dripping is observed; pinching can only happen if additional effects such as van der Waals forces are included. It is useful to compare the critical value of $\epsilon$ found for dripping, with the capillary length $\ell_{c}=\sqrt{\sigma_{12}^{*} / \rho_{2}^{*} g^{*}}$. Taking layer 1 to be air, i.e., $\rho_{1}^{*} \approx 1.225 \mathrm{~kg} / \mathrm{m}^{3}$, and taking parameters as in the DNS performed in this section, we have the density of the liquid layer region 2 being $\rho_{2}^{*}=r_{1} \rho_{1}^{*} \approx 98 \mathrm{~kg} / \mathrm{m}^{3}$. In addition, assuming a surface tension coefficient $\sigma_{12}^{*}=72 \times 10^{-3} \mathrm{~N} / \mathrm{m}$ (this is a typical value for water-air interfaces), we find $\ell_{c} \approx 8.6 \mathrm{~mm}$. The value of $L_{D}^{*}$ can be found from the dimensionless surface tension formula $\sigma=\sigma_{12}^{*} / \rho_{2}^{*} g^{*} L_{D}^{* 2}$, which was set to $\sigma=0.012665$ in the present computations. We find $L_{D} \approx 7.7 \mathrm{~cm}$ and since the critical value of $\epsilon$ that gives dripping is between $0.45 / 2 \pi$ and $0.5 / 2 \pi$, it follows that its dimensional thickness is approximately $6 \mathrm{~mm}$, which is consistent with the capillary length estimated earlier.

\section{APPLICATION: ACTIVE CONTROL OF THE RAYLEIGH-TAYLOR INSTABILITY}

In what follows we use a time-dependent electric field to produce controlled interfacial oscillations, with no moving mechanical parts, with possible implications for mixing at small scales. 


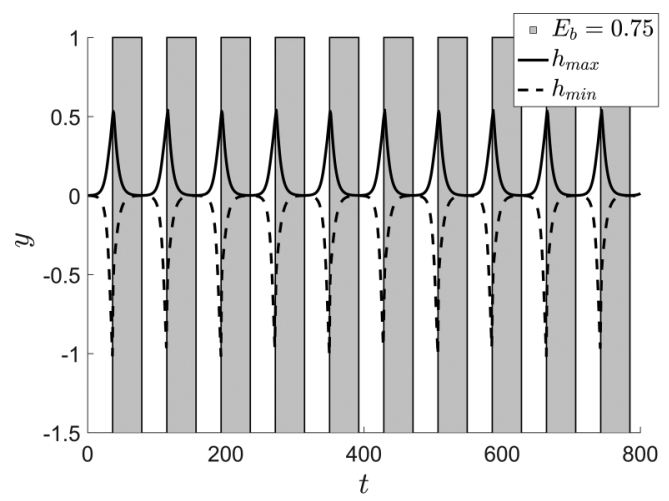

(a)

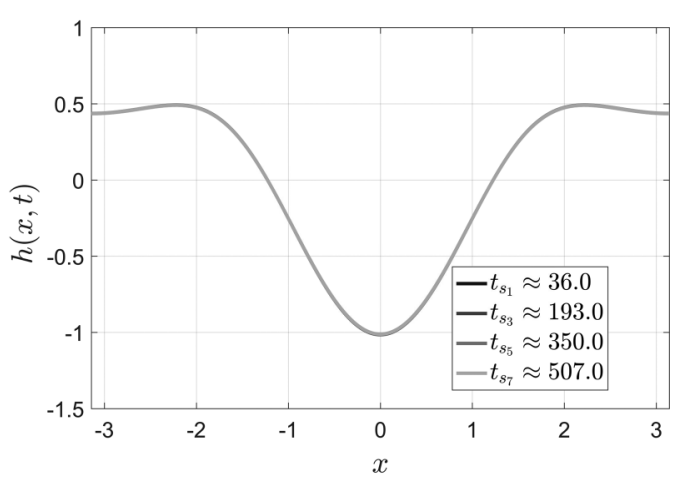

(b)

FIG. 17. On-off electric field with the electric field activated when $h_{\max }$ exceeds the threshold value $y_{t}=0.5$ and switched off when the interface maximum reaches the same level as in the initial condition: (a) evolution of interfacial minimum $h_{\min }$ and maximum $h_{\max }$ in time and (b) interfacial shapes immediately prior to switching on the electric field in four different cycles.

Simple control protocols have already been investigated in a geometry of infinite vertical extent [18] and then tailored towards mixing studies in two and three dimensions [24] when the walls were placed far away from the undisturbed position of the interface. The latter study uses vertical electric fields to introduce instability in otherwise stably stratified flows; here we use horizontal electric fields to arrest gravitational instabilities. Results using both the long-wave model and DNS will be presented.

To fix matters we solve the evolution equation (41) with the same parameters as in case (b) in Sec. III, i.e., $W_{e}=0.35, \epsilon_{p}=3.0$, and $\epsilon_{B}=\epsilon_{T}=1.5$. The electric field strength $E_{b}$ is chosen to alternate between 0 and 0.75 , which is above the stability threshold predicted by linear theory and is selected to induce stabilization even when outside the linear regime.

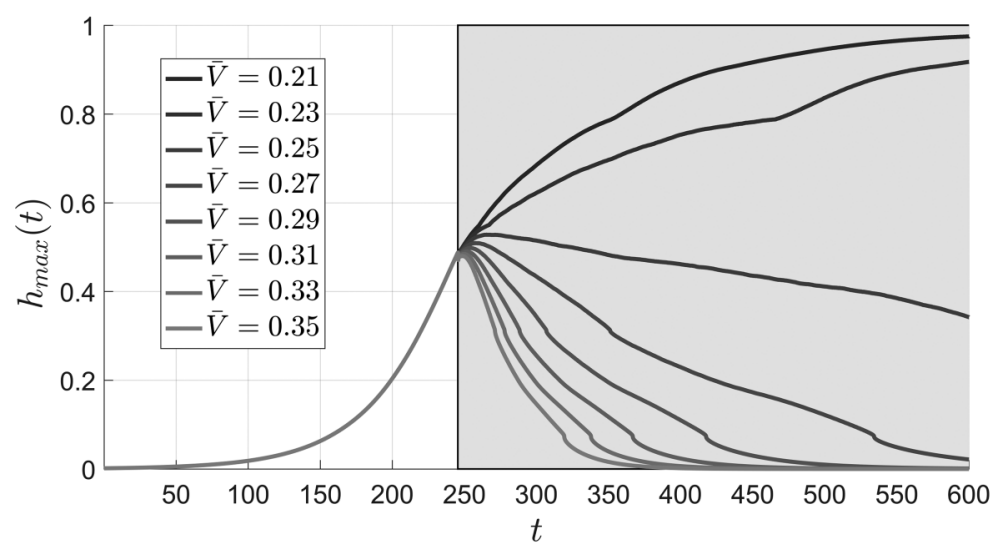

(a)

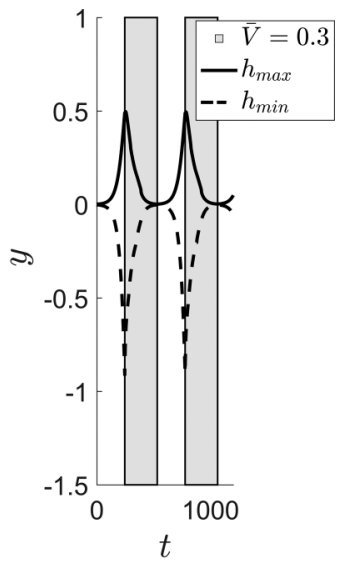

(b)

FIG. 18. On-off electric field with the electric field activated when $h_{\max }$ exceeds the threshold value $y_{t}=0.5$ and switched off when the interface maximum reaches the same level as in the initial condition. (a) Interface maximum as a function of time for different values of $\bar{V}$ when switching the electric field on at $t=245.5$ (single cycle). (b) The evolution of the interfacial extrema $h_{\max }(t)$ and $h_{\min }(t)$ is compared to the dynamics obtained in the equivalent configuration in the long-wave model, presented previously in Fig. 17(a). 


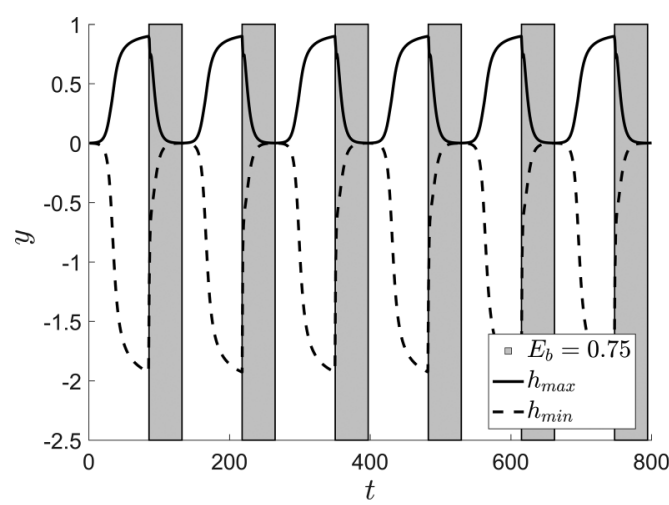

(a)

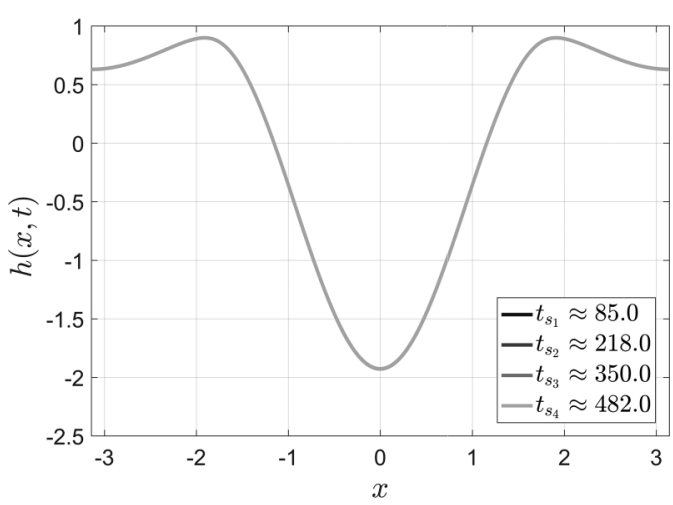

(b)

FIG. 19. On-off electric field with the electric field activated when $h_{\max }$ exceeds the threshold value $y_{t}=0.9$ and switched off when the interface maximum reaches the same level as in the initial condition: (a) evolution of interfacial minimum $h_{\min }$ and maximum $h_{\max }$ in time and (b) interfacial shapes immediately prior to switching on the electric field in four different cycles.

The suggested mechanism is summarized as follows. Starting from a $k=1$ initial perturbation and an initial amplitude of $5 \times 10^{-4}$, we allow the Rayleigh-Taylor instability to evolve naturally until a certain threshold level $y_{t}$ is reached by the interfacial maximum $h_{\max }(t)$. As soon as $y_{t}$ is exceeded, the electric field is switched on and, after an initial transient, a strong stabilization occurs that, if sustained, would ultimately drive the interface to a flat state. Before this happens, however, we switch the electric field off as soon as $h_{\max }(t)$ drops below its initial amplitude and repeat this cycle over several periods.

Figure 17(a) shows the evolution of the interfacial extrema $h_{\min }(t)$ (dashed curve) and $h_{\max }(t)$ (solid curve) over the duration of the nonlinear calculation. The background is colored in white when the electric field is switched off, allowing the instability to grow over tens of time units. Once $h_{\max }$ reaches the threshold level, the electric field is switched on as illustrated with a dark gray background. Under the stabilizing action of the field, both extrema decrease to their initial amplitudes as seen in Fig. 17(a). A threshold value $y_{t}=0.5$ is sufficient for nonlinear effects to emerge and Fig. 17(b) presents the interfacial shapes one time step before the electric field is switched on. The results strongly suggest a robust time periodicity of the phenomena. The duration of the periods when the field is off is $t_{\text {off }} \approx 36.26$, while the on periods are slightly smaller with $t_{\text {on }} \approx 32.7$. The interfacial shapes are virtually indistinguishable between consecutive on-off cycles. The robustness of the dynamics suggests that we can repeat the oscillations over several tens or hundreds of cycles to reach competitive mixing designs. We also note that even though a time-dependent electric field is proposed in the context of a quasistatic approximation, the scales involved ensure the validity of such an approach (see Appendix B of [18] for details).

The sustained oscillations are also faithfully reproduced in the direct numerical simulations. We use the same parameter values as in the second test case in Sec. IV B. For the parameters of Figs. 17(a) and 17(b), the equivalent stabilizing voltage to be imposed is $\bar{V} \approx 0.3$. Results with a number of different values of $\bar{V}$ are illustrated in Fig. 18(a); it can be seen that voltages $\bar{V}>0.25$ stabilize the interface and return it to its almost flat state by the end of the computation. For values of $\bar{V} \approx 0.3$ the relative duration of off and on cycles as well as shapes are in good agreement between the long-wave model and the DNS.

Next we turn to a more stringent threshold $y_{t}=0.9$ that allows the interface to get closer to the wall before the field is turned on to cause stabilization and sustained oscillations. We proceed with computations based on the model (41) due to the prohibitive cost of the DNS in this case. Figures 19(a) and 19(b) indicate that once again the on-off cycles are sustained robustly even in 
this more challenging regime. Here the off periods increase from 36.25 time units to approximately 85.64 time units in order to accommodate the growth closer to the wall. By comparison, the on periods only require a mild increase in order to steer the interface back to its initial perturbation.

\section{CONCLUSION}

This study derived and used model long-wave equations as well as direct numerical simulations to study the effect of horizontal electric fields on the Rayleigh-Taylor instability of viscous dielectric fluids. Small-scale channels are typically enclosed within polymer slabs and our model took into account the electrostatics in these slabs and their electrohydrodynamic coupling with the fluids inside the channel. The analysis leading to Eq. (41) was carried out for bounding slabs of infinite extent and this is appropriate since the channel thickness is typically smaller than that of the slabs. The theory can be modified in a straightforward way for slabs of finite thickness, at the expense of more complicated Fourier symbols of the nonlocal term in Eq. (41). In addition, direct numerical simulations that necessarily use finite slab geometries were found to be in excellent agreement with the model. We also demonstrated the possibility of using the imposed electric field as an active control parameter to induce sustained time-periodic nonlinear oscillations of the interface that may have relevance in mixing in small-scale geometries (see [24] for related approaches).

There are several research directions that can be pursued based on this work, including the effect of topographical structures (e.g., of finite extent for simplicity) on the wall wetted by the liquid layer as well as the addition of pressure-driven flow as encountered in microfluidic devices, for instance. In the former case the effect of wall topography will induce a nonuniform field locally and hence nonuniform base states as opposed to the flat ones studied here. Adding flow coupled with horizontal field stabilization is expected to produce active-dissipative dynamics reminiscent of the Kuramoto-Sivashinsky equation, for example.

\section{ACKNOWLEDGMENT}

The work of R.C. and D.T.P. was supported by the Engineering and Physical Sciences Research Council under Grants No. EP/K041134/1 and No. EP/L020564/1.

\section{APPENDIX: NUMERICAL METHOD FOR THE THIN-FILM EQUATION}

We consider the problem of numerically solving (41) in the form

$$
H_{t}+\left[f_{1}(H) H_{x x x}\right]_{x}+\left[f_{2}(H)\right]_{x x}+\left[f_{3}(H)\{B[H](x)\}_{x x}\right]_{x}=0,
$$

where $B[H](x)$ is the nonlocal term due to the electric field known in Fourier space as (40) and the polynomial functions $f_{i}(H), i=1,2,3$, are as follows:

$$
f_{1}(H)=\frac{W_{e}}{3}(1-H)^{3}, \quad f_{2}(H)=-\frac{1}{12}(1-H)^{4}, \quad f_{3}(H)=\frac{E_{b}\left(1-\epsilon_{p}\right)}{3}(1-H)^{3},
$$

by discretizing it on a periodic domain $x \in[-L, L]$ using the finite-difference method presented in Ref. [20] and modified as described below in order to include the new electric field term, $\left[\left\{f_{3}(H) B[H](x)\right\}_{x x}\right]_{x}$. We first briefly describe the discretization of the fluid part $\left(E_{b}=0\right)$ of (A1) with second-order accurate finite differencing on a fixed grid $x_{m}=(m-M) \Delta x, m=1,2, \ldots, 2 M$, $\Delta x=L / M$. Using the convention $x_{m+1 / 2}=\left(x_{m}+x_{m+1}\right) / 2$, we obtain the system of ordinary differential equations

$$
\frac{d H_{m}}{d t}+\frac{f_{1}\left(H_{m+1 / 2}\right) \partial_{3}(H)_{m+1 / 2}-f_{1}\left(H_{m-1 / 2}\right) \partial_{3}(H)_{m-1 / 2}}{\Delta x}+\partial_{2}\left[f_{2}(H)\right]_{m}=0
$$

where we note that $\partial_{i}$ represents a standard second-order-accurate finite-difference approximation to the $i$ th spatial derivative. The system of ordinary differential equations (A3) is discretized in time 
with the second-order-accurate Crank-Nicolson scheme as

$$
\mathbf{H}^{n+1}+\frac{\Delta t}{2} \mathbf{F}^{\text {fluid }}\left(\mathbf{H}^{n+1}\right)=\mathbf{H}^{n}-\frac{\Delta t}{2} \mathbf{F}^{\text {fluid }}\left(\mathbf{H}^{n}\right)
$$

and at every time step we solve a nonlinear algebraic system for $\mathbf{H}^{n+1}$ with Newton iteration for which the Jacobian matrix is

$$
\mathbb{J}=\mathbb{I}+\frac{\Delta t}{2} \frac{\partial \mathbf{F}^{\text {fluid }}}{\partial \mathbf{H}},
$$

where the elements of the $\frac{\partial \mathbf{F}^{\text {fluid }}}{\partial \mathbf{H}}$ matrix are known explicitly.

Next our modification of the scheme in Ref. [20] is described by considering the evolution PDE

$$
H_{t}+\left[f_{3}(H)\{B[H](x)\}_{x x}\right]_{x}=0
$$

with periodic boundary conditions. We let $\mathbf{H}^{n}$ be the discretization of $H$ at time $t_{n}$ on the computational grid. The Crank-Nicolson discretization in time is then, using the product rule,

$$
\frac{\mathbf{H}^{n+1}-\mathbf{H}^{n}}{\Delta t}+\frac{1}{2}\left[\mathbf{f}_{3}^{\prime}(\mathbf{h})\left(D_{x} \mathbf{H}\right) B_{x x}+\mathbf{f}_{3}(\mathbf{H}) B_{x x x}\right]^{n+1}+\frac{1}{2}\left[\mathbf{f}_{3}^{\prime}(\mathbf{H})\left(D_{x} \mathbf{H}\right) B_{x x}+\mathbf{f}_{3}(\mathbf{H}) B_{x x x}\right]^{n}=0
$$

where the vector-vector products are computed pointwise and the $D_{x}$ operator is the discrete differentiation operator. We now need a method for calculating the Jacobian of the function evaluations and we cannot use the fast Fourier transform to compute the nonlocal term. We thus rewrite the equation above in the following way:

$$
\mathbf{H}^{n+1}+\frac{\Delta t}{2}\left[\mathbf{f}_{3}^{\prime}(\mathbf{H})(\mathbb{D} \mathbf{H})(\mathbb{G} \mathbf{H})+\mathbf{f}_{3}(\mathbf{H})(\tilde{\mathbb{G}} \mathbf{H})\right]^{n+1}=-\frac{\Delta t}{2}\left[\mathbf{f}_{3}^{\prime}(\mathbf{H})(\mathbb{D} \mathbf{H})(\mathbb{G} \mathbf{H})+\mathbf{f}_{3}(\mathbf{H})(\tilde{\mathbb{G}} \mathbf{H})\right]^{n},
$$

where we made the operator substitutions in notation $D_{x}=: \mathbb{D}, B_{x x}=: \mathbb{G} \mathbf{H}$, and $B_{x x x}=: \tilde{\mathbb{G}} \mathbf{H}$. We identify the bracketed term on the left-hand side of the above equation with $\mathbf{F}^{\text {electric }}\left(\mathbf{H}^{n+1}\right)$ and see that we now have a nonlinear system of equations to solve and need an explicit formula for $\frac{\partial \mathbf{F}^{\text {electric }}}{\partial \mathbf{H}}$ to perform Newton iteration. To this end we denote the discrete Fourier transform matrix by $\mathbb{M}_{F}^{\partial \mathbf{H}}, k$ is the wave number, and write out our operators in matrix form as

$$
\begin{array}{ll}
\mathbb{G}:=\mathbb{M}_{F}^{-1} \Sigma_{1} \mathbb{M}_{F}, \quad \Sigma_{1}:=\operatorname{diag}\left[-k^{2} \lambda(k)\right], \\
\mathbb{D}:=\mathbb{M}_{F}^{-1} \Sigma_{2} \mathbb{M}_{F}, \quad \Sigma_{2}:=\operatorname{diag}[i k \lambda(k)], \\
\tilde{\mathbb{G}}:=\mathbb{M}_{F}^{-1} \Sigma_{3} \mathbb{M}_{F}, \quad \Sigma_{3}:=\operatorname{diag}\left[-i k^{3} \lambda(k)\right],
\end{array}
$$

and define $\mathbf{d}_{i}, \mathbf{g}_{i}$, and $\tilde{\mathbf{g}}_{i}$ as the $i$ th row of $\mathbb{D}, \mathbb{G}$, and $\widetilde{\mathbb{G}}$, respectively, where

$$
\lambda(k)=i\left(1-\epsilon_{p}\right) \frac{k \cosh (k)+\epsilon_{B}|k| \sinh (k)}{\left(\epsilon_{B}+\epsilon_{T}\right)|k| \cosh (k)+\left(1+\epsilon_{B} \epsilon_{T}\right) k \sinh (k)}
$$

is the symbol in Fourier space of the convolution kernel deduced from (40). Using this notation, we derive the following relationships for the elements of the Jacobian $\mathbb{J}$ :

$$
\begin{aligned}
\frac{\partial \mathbf{F}_{m}^{\text {electric }}}{\partial \mathbf{H}_{j}}= & \frac{\Delta t}{2}\left[f_{3}^{\prime}\left(H_{m}\right) \mathbb{D}_{m j}\left(\mathbf{g}_{m} \cdot \mathbf{H}\right)+f_{3}^{\prime}\left(h_{m}\right)\left(\mathbf{d}_{m} \cdot \mathbf{H}\right) \mathbb{G}_{m j}+f_{3}\left(H_{m}\right) \tilde{\mathbb{G}}_{m j}\right], \quad m \neq j \\
\frac{\partial \mathbf{F}_{m}^{\text {electric }}}{\partial \mathbf{H}_{j}}= & 1+\frac{\Delta t}{2}\left[f_{3}^{\prime \prime}\left(H_{m}\right)\left(\mathbf{d}_{m} \cdot \mathbf{H}\right)\left(\mathbf{g}_{m} \cdot \mathbf{H}\right)+f_{3}^{\prime}\left(h_{m}\right) \mathbb{D}_{m j}\left(\mathbf{g}_{m} \cdot \mathbf{H}\right)\right. \\
& \left.+f_{3}^{\prime}\left(H_{m}\right)\left(\mathbf{d}_{m} \cdot \mathbf{H}\right) \mathbb{G}_{m j}+f_{3}^{\prime}\left(H_{m}\right)\left(\tilde{\mathbf{g}}_{m} \cdot \mathbf{H}\right)+f_{3}\left(H_{m}\right) \tilde{\mathbb{G}}_{m j}\right], \quad m=j .
\end{aligned}
$$

Adding the matrix (A9) to the $\frac{\partial \mathbf{F}^{\text {fuid }}}{\partial \mathbf{H}}$ matrix allows us to perform Newton iterations to solve for $\mathbf{H}^{n+1}$ in the presence of both the fluid and the electric field terms. 
In our implementation we refine the spatial grid whenever a finer discretization is detected to be required in order to accurately resolve finer features of the evolving solution. Specifically, we take a Fourier transform of the solution in each time step and examine the magnitudes of the Fourier modes. When more than a threshold, for example, $\frac{2}{3}$ of the Fourier modes are larger than a tolerance $\epsilon_{x}$ we double the number of grid points. Note that this implies we are implementing global adaptivity, not adaptivity only in regions with finer features. Implementing our upscaling method is straightforward with Fourier interpolation; we take our Fourier transformed solution $\hat{\mathbf{h}}_{M}$ on a grid with $M$ grid points, pad $M$ higher wave numbers with zero, and then transform back to obtain a solution $\mathbf{h}_{2 M}$ defined on $2 M$ grid points. We also control the time step by employing a local error indicator $e_{m}$, which approximates $\frac{\Delta t_{n-1}}{H_{m}^{n}} \frac{d^{2} H_{m}^{n}}{d t^{2}}[20,25]$ :

$$
e_{m}=\frac{2 \Delta t_{n-1}}{\Delta t_{n-2}} \frac{\Delta t_{n-2} H_{m}^{n+1}+\Delta t_{n-1} H_{m}^{n-1}-\left(\Delta t_{n-2}+\Delta t_{n-1}\right) H_{m}^{n}}{\left(\Delta t_{n-2}+\Delta t_{n-1}\right) H_{m}^{n}} .
$$

We increase $\Delta t$ by $10 \%$ whenever $e_{m}<\frac{3}{4} \epsilon_{t}$ for all $m$ and decrease it when $e_{m}>\epsilon_{t}$, where $e_{t}$ is the time accuracy to be maintained throughout the computation time interval. For solving the linear systems in the nonlinear Newton iterations, we additionally use a GMRES solver with a preconditioner derived from the linearized form of Eq. (A1) [26].

[1] S. G. Yiantsios and B. G. Higgins, Rayleigh-Taylor instability in thin viscous films, Phys. Fluids A 1, 1484 (1989).

[2] L. A. Newhouse and C. Pozrikidis, The Rayleigh-Taylor instability of a viscous liquid layer resting on a plane wall, J. Fluid Mech. 217, 615 (2006).

[3] A. Egowainy and N. Ashgriz, The Rayleigh-Taylor instability of viscous fluid layers, Phys. Fluids 9, 1635 (1997).

[4] P. S. Hammond, Nonlinear adjustment of a thin annular film of viscous fluid surrounding a thread of another within a circular cylindrical pipe, J. Fluid Mech. 137, 363 (1983).

[5] J. R. Lister, J. M. Rallison, A. A. King, L. J. Cummings, and O. E. Jensen, Capillary drainage of an annular film: The dynamics of collars and lobes, J. Fluid Mech. 552, 311 (2006).

[6] L. Limat, P. Jenffer, B. Dagens, M. Fermigier, and J. E. Wesfreid, Gravitational instabilities of thin liquid layers: Dynamics of pattern selection, Physica D 61, 166 (1992).

[7] M. Fermigier, L. Limat, J. E. Wesfreid, P. Boudinet, and C. Quillet, Two-dimensional patterns in RayleighTaylor instability of a thin layer, J. Fluid Mech. 236, 349 (1992).

[8] J. R. Lister, J. M. Rallison, and S. J. Rees, The nonlinear dynamics of pendent drops on a thin film coating the underside of a ceiling, J. Fluid Mech. 647, 239 (2010).

[9] A. J. Babchin, A. L. Frenkel, B. G. Levich, and G. I. Sivashinsky, Nonlinear saturation of Rayleigh-Taylor instability in thin films, Phys. Fluids 26, 3159 (1983).

[10] D. Halpern and A. L. Frenkel, Saturated Rayleigh-Taylor instability of an oscillating Couette film flow, J. Fluid Mech. 446, 67 (2001).

[11] O. Haimovich and A. Oron, Nonlinear dynamics of a thin liquid film on an axially oscillating cylindrical surface, Phys. Fluids 22, 032101 (2010).

[12] J. R. Melcher, Electrohydrodynamic and magnetohydrodynamic surface waves and instability, Phys. Fluids 4, 1348 (1961).

[13] J. R. Melcher, Field-Coupled Surface Waves (Technology Press, Cambridge, 1963).

[14] N. T. Eldabe, Effect of a tangential electric field on Rayleigh-Taylor instability, J. Phys. Soc. Jpn. 58, 115 (1989).

[15] A. Joshi, M. C. Radhakrishna, and N. Rudraiah, Rayleigh-Taylor instability in dielectric fluids, Phys. Fluids 22, 064102 (2010).

[16] L. L. Barannyk, D. T. Papageorgiou, and P. G. Petropoulos, Suppression of Rayleigh-Taylor instability using electric fields, Math. Comput. Simul. 82, 1008 (2010). 
[17] L. L. Barannyk, D. T. Papageorgiou, P. G. Petropoulos, and J.-M. Vanden-Broeck, Nonlinear dynamics and wall touch-up in unstably stratified multilayer flows under the action of electric fields, SIAM J. Appl. Math. 75, 92 (2015).

[18] R. Cimpeanu, D. T. Papageorgiou, and P. G. Petropoulos, On the control and suppression of the RayleighTaylor instability using electric fields, Phys. Fluids 26, 022105 (2014).

[19] D. T. Papageorgiou and J.-M. Vanden-Broeck, Large-amplitude capillary waves in electrified fluid sheets, J. Fluid Mech. 508, 71 (2004).

[20] D. Tseluiko and D. T. Papageorgiou, Nonlinear dynamics of electrified thin liquid films, SIAM J. Appl. Math. 67, 1310 (2007).

[21] A. F. Jones and S. D. R. Wilson, The film drainage problem in droplet coalescence, J. Fluid Mech. 87, 263 (1978).

[22] S. Popinet, Gerris: A tree-based adaptive solver for the incompressible Euler equations in complex geometries, J. Comput. Phys. 190, 572 (2003).

[23] S. Popinet, An accurate adaptive solver for surface-tension-driven interfacial flows, J. Comput. Phys. 228, 5838 (2009).

[24] R. Cimpeanu and D. T. Papageorgiou, Electrostatically induced mixing in confined stratified multi-fluid systems, Int. J. Multiphase Flow 75, 194 (2015).

[25] A. L. Bertozzi and M. Pugh, The lubrication approximation for thin viscous films: The moving contact line with a 'porous media' cut-off of van der Waals interactions, Nonlinearity 7, 1535 (1994).

[26] L. S. Mulholland and D. M. Sloan, The role of preconditioning in the solution of evolutionary partial differential equations by implicit Fourier pseudospectral methods, J. Comput. Appl. Math. 42, 157 (1992). 